Draft version OCtober 26, 2016

Preprint typeset using $\mathrm{IAT}_{\mathrm{E}} \mathrm{X}$ style emulateapj v. 5/2/11

\title{
X-RAY REFLECTION AND AN EXCEPTIONALLY LONG THERMONUCLEAR HELIUM BURST FROM IGR J17062-6143
}

\author{
L. Keek, ${ }^{1,2}$ W. Iwakiri, ${ }^{3}$ M. Serino, ${ }^{3}$ D. R. Ballantyne, ${ }^{4}$ J. J. M. in 'T Zand, ${ }^{5}$ T. E. Strohmayer ${ }^{1}$ \\ Draft version October 26, 2016
}

\begin{abstract}
Thermonuclear X-ray bursts from accreting neutron stars power brief but strong irradiation of their surroundings, providing a unique way to study accretion physics. We analyze $M A X I /$ GSC and Swift/XRT spectra of an exceptionally long flash observed from IGR J17062-6143 in 2015. It is a rare case of recurring bursts at the low accretion luminosity of $0.15 \%$ Eddington. Spectra from MAXI, Chandra, and NuSTAR observations taken between the 2015 burst and the previous one in 2012 are used to determine the accretion column. We find it to be consistent with the burst ignition column of $5 \times 10^{10} \mathrm{~g} \mathrm{~cm}^{-2}$, which indicates that it is likely powered by burning in a deep helium layer. The burst flux is observed for hours, and decays as a straight power law: $F \propto t^{-1.15}$. The burst and persistent spectra are well described by thermal emission from the neutron star, Comptonization of this emission in a hot optically thin medium surrounding the star, and reflection off the photoionized accretion disk. At the burst peak, the Comptonized component disappears, when the burst may dissipate the Comptonizing gas, and it returns in the burst tail. The reflection signal indicates that the inner disk is truncated at $\sim 10^{2} R_{\mathrm{g}}$ before the burst, but moves closer to the star during the burst. Subject headings: accretion, accretion disks — stars: neutron — stars: individual: IGR J17062-6143 - X-rays: binaries - X-rays: bursts
\end{abstract}

\section{INTRODUCTION}

Mass transfer from a binary companion star onto a neutron star can enrich the latter's surface in hydrogen and/or helium. The strong compression by the neutron star's gravity induces nuclear fusion in the surface layer. If the nuclear burning proceeds as a thermonuclear runaway, the accreted material burns within seconds, powering a bright Type I X-ray burst (Grindlav et al. 1976; Belian et al. 1976; Woosley \& Taam 1976; Maraschi \& Cavaliere 1977). Most of the thousands of observed bursts last $\sim 10-100 \mathrm{~s}$ (e.g., Cornelisse et al. 2003; Galloway et al. 2008). Intermediate duration bursts $(\sim 100-1000 \mathrm{~s})$ and superbursts $(\gtrsim 1000 \mathrm{~s})$ are observed relatively rarely. They are thought to be powered by the unstable burning of deep layers of helium and carbon, respectively (e.g., Keek \& in 't Zand 2008). Several tens of bursts have been detected from both categories, with durations of minutes to hours that allow for higher quality spectra to be collected. For example, for two superbursts reflection features were detected (Strohmaver \& Brown 2002; Ballantyne \& Strohmaver 2004; Keek et al. 2014b), and several intermediate duration bursts exhibit superexpansion and "achromatic" variability (in 't Zand et al. 2011; Degenaar et al. 2013). These are instances where X-ray

\footnotetext{
laurens.keek@nasa.gov

${ }^{1}$ X-ray Astrophysics Laboratory, Astrophysics Science Division, NASA/GSFC, Greenbelt, MD 20771

2 CRESST and the Department of Astronomy, University of Maryland, College Park, MD 20742

3 MAXI team, RIKEN, 2-1 Hirosawa, Wako, Saitama 3510198, Japan

4 Center for Relativistic Astrophysics, School of Physics, Georgia Institute of Technology, 837 State Street, Atlanta, GA 30332 0430

5 SRON Netherlands Institute for Space Research, Sorbonnelaan 2, 3584 CA Utrecht, The Netherlands
}

bursts have a strong impact on the accretion environment around the neutron star, and demonstrate how Xray bursts can be employed to study accretion physics (e.g., Ballantyne \& Everett 2005).

The majority of bursts has been observed with instruments sensitive above $\sim 2 \mathrm{keV}$ and with modest spectral resolution. The burst signal in the soft Xray band is relatively unexplored, even though, for instance, the burst reflection signal may dominate this band (Ballantyne 2004). A bright burst observed from IGR J17062-6143 in 2012 with the Swift observatory exhibited spectral emission features below $2 \mathrm{keV}$. Robotic optical telescopes detected a declining R-band flux in the 2 hours following the Swift/BAT trigger (Ivarsen et al. 2012; Meehan et al. 2012). On 11/3/2015 the nova alert system (Negoro et al. 2016) of the Monitor of All-sky $X$-ray Image (MAXI) detected an even more powerful burst from this source (Negoro et al. 2015). A campaign of follow-up observations was subsequently performed by Swift (Iwakiri et al. 2015). In this paper we analyze the observations of the 2015 burst to investigate its impact on its surroundings.

IGR J17062-6143 was discovered in 2006 at the start of an outburst that lasted several years (Churazov et al. 2007; Ricci et al. 2008; Remillard \& Levine 2008). Since then it has continued to be active at a low flux. Chandra gratings spectra of the persistent emission exhibit emission and absorption lines (Degenaar et al. 2016). NuS$T A R$ observations revealed reflection features, that indicate that the accretion disk is highly ionized and truncated far from the neutron star surface. Degenaar et al. (2016) discuss how a strong magnetic field could truncate the disk, form a radiatively inefficient accretion flow, and act as a propeller to drive an outflow (for a review see D'Angelo et al. 2015). However, our knowledge of IGR J17062-6143 remains limited. The neutron star 
TABLE 1

Burst Observations

\begin{tabular}{cccc}
\hline Instrument & ObsID & Mode & Exposure $(\mathrm{ks})$ \\
\hline MAXI/GSC & $11 / 3 / 201510: 29$ UT & & 0.06 \\
& $11 / 3 / 201512: 03$ UT & & 0.06 \\
\hline Swift/XRT & 00037808006 & PC & 1.7 \\
& 00037808008 & WT & 3.4 \\
& 00037808008 & PC & 1.8 \\
& 00037808009 & PC & 5.6 \\
& 00037808010 & WT & 4.0 \\
& 00037808012 & WT & 0.9 \\
& 00037808015 & WT & 6.2 \\
00037808016 & WT & 6.7 \\
& 00037808017 & WT & 6.9 \\
& 00037808018 & WT & 6.7 \\
& 00037808019 & WT & 7.2 \\
& 00037808020 & WT & 4.3 \\
& 00037808021 & PC & 4.5 \\
& 00037808022 & PC & 5.0 \\
& 00037808023 & PC & 3.9 \\
& 00037808024 & PC & 6.5 \\
\hline
\end{tabular}

spin, the composition and inclination of the disk, and the binary period are unknown. Intermediate duration bursts and accretion at a constant low rate have been associated with Ultra Compact X-ray Binaries (UCXBs; e.g., in 't Zand et al. 2007). Therefore, our working assumption is that IGR J17062-6143 is a UCXB where helium-rich material is accreted.

After describing the employed observations and spectral models (Section 2), we jointly analyze the persistent MAXI, Chandra, and NuSTAR spectra to determine the time-averaged persistent flux between the two bursts (Section 3). The MAXI and Swift burst spectra are analyzed to establish the properties of the thermonuclear flash (Section 4). In both cases we fit a simple phenomenological model as well as a more physically motivated model that includes Comptonization and photoionized reflection. The results show that this burst had a strong influence on the accretion geometry (Section 5), and we conclude that it is one of the most powerful helium flashes ever observed from an accreting neutron star (Section 6).

\section{OBSERVATIONS AND SPECTRAL MODELS}

\subsection{Burst Observations}

MAXI (Matsuoka et al. 2009) was installed on the International Space Station (ISS) in 2009. We employ data from the Gas Slit Camera (GSC; Mihara et al. 2011; Sugizaki et al. 2011), which consists of 12 xenon-filled proportional counters that are sensitive in the $2-30 \mathrm{keV}$ energy range and have a combined collecting area of $5350 \mathrm{~cm}^{2}$. A slit and slat collimator restricts the field of view to a narrow elongated region of $3^{\circ} \times 80^{\circ}$. $85 \%$ of the sky is scanned each 92 minute orbit of the ISS. We extract source spectra for the triggering GSC scan on 11/3/2015 10:29 UT as well as the subsequent scan (Table 1). The source is visible for one minute during each scan, and the effective area peaks at $3 \mathrm{~cm}^{2}$ in the middle of the scans. The first spectrum has 893 counts, whereas the second consists of only 90 counts. The background and instrument response are modeled with tools provided by the instrument team (Sugizaki et al. 2011).

The Swift observatory was launched in 2004 (Gehrels et al. 2004). Its main pointed X-ray instru-
TABLE 2

Observations of Persistent Emission

\begin{tabular}{ccc}
\hline Instrument & ObsID & Exposure $(\mathrm{ks})$ \\
\hline MAXI/GSC & $6 / 26 / 2012-11 / 2 / 2015$ & $2,302.5$ \\
\hline Chandra $/$ HETGS & $15749(10 / 25 / 2014)$ & 29.3 \\
& $17543(10 / 27 / 2014)$ & 63.5 \\
\hline NuSTAR & $30101034002(05 / 06 / 2015)$ & 70.1 \\
\hline
\end{tabular}

ment is the X-Ray Telescope (XRT; Burrows et al. 2005). The XRT is a CCD imager sensitive in the $0.2-10 \mathrm{keV}$ band with an effective area of $120 \mathrm{~cm}^{2}$ at $1.5 \mathrm{keV}$. Swift's other pointed instrument is the Ultraviolet/Optical Telescope (UVOT; Roming et al. 2005), which records CCD images in the $170-650 \mathrm{~nm}$ wavelength range. UVOT has 7 filters to select a narrow wavelength interval from this range. Starting 3 hours after the first $M A X I / G S C$ scan, Swift performed a series of pointed observations of IGR J17062-6143 (Iwakiri et al. 2015). A total of $75.3 \mathrm{ks}$ were collected over 11.7 days (Table 1. Figure 1). The XRT observations were performed in either Photon Counting (PC) mode or Windowed Timing (WT) mode. Whereas in PC mode the full CCD image is stored, in WT mode a reduced $1 \mathrm{~d}$ image is read out to increase the time resolution. We use XRTPIPELINE to extract spectra in the $0.5-10 \mathrm{keV}$ band and light curves from a circular region with a radius of 70.8 arcsec (30 pixels) centered on the source, and from an off-source location as background. The standard selection of event grades are used: $0-12$ for PC mode and $0-2$ for WT mode. The PC mode data suffer from pile-up. We exclude the piled-up center of the point-spread-function. The size of this region is determined by comparing the observed spatial distribution of events to a King profile that describes the expected point-spread-function (Moretti et al. 2005): the excluded regions have a radius ranging from 20 arcsec at the highest flux to 9 arcsec at the lowest flux. The detector light curves are inspected for background flares. The ancillary response is generated by XRTPIPELINE, and we employ the appropriate response matrices provided by the instrument team. The data are labeled with an Observation Identifier (ObsID), and each ObsID represents several consecutive satellite orbits. For the first 2 ObsIDs, which cover the initial burst decay, we create separate spectra for each orbit. For the rest, one spectrum per ObsID is generated. The resulting XRT spectra each have between 840 and 13175 counts, with a median value of 5003 counts. In Section 4.3 we demonstrate that these spectra provide sufficient time resolution to resolve the cooling trend of the burst.

\subsection{Observations of Persistent Emission}

Swift's Burst Alert Telescope (BAT; Barthelmy et al. 2005) is a coded-mask imager with a wide field of view of $1.4 \mathrm{sr}$ and a $15-150 \mathrm{keV}$ band pass. We employ BAT in combination with MAXI to illustrate the long term evolution of the persistent flux (Figure 2). The source was first detected at the onset of its outburst in 2006, and since 2010 it has been accreting continuously at a low rate. MAXI operations started in 2009. We are especially interested in the period between the burst observed in 2012 and the one in 2015 (studied in this paper). To quantify the variability in that time interval, we take the root mean squared of the photon flux at a 70 day resolution: it is $38 \%$ of the mean for BAT and 


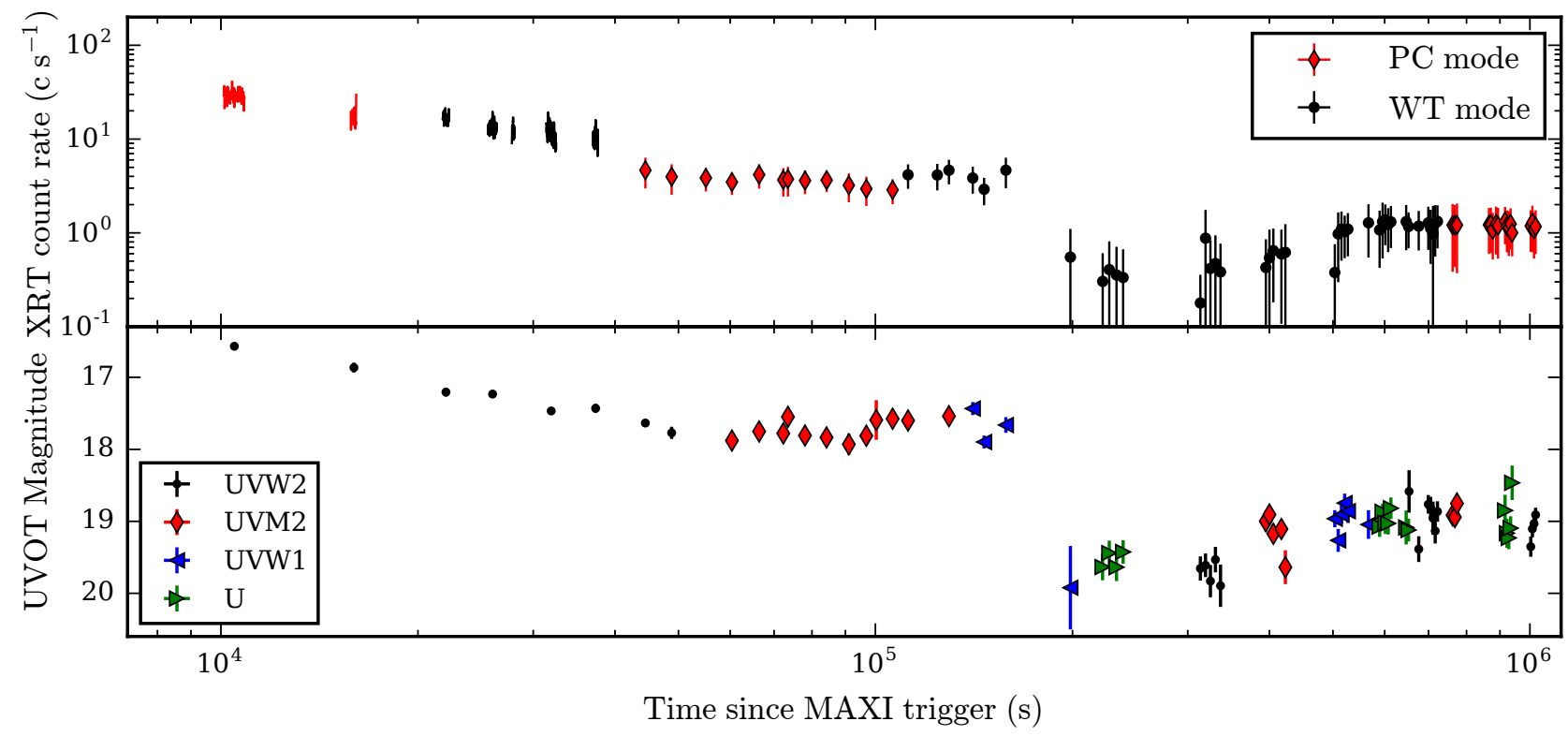

FIG. 1.- (Top) Light curve of the XRT count rates as a function of time since the MAXI burst detection. For the first 11 hr we show the data at $10 \mathrm{~s}$ time resolution, whereas for later observations each data point is the average per orbit and error bars indicate the root-mean-squared of the data at 10 s resolution. The first 2 orbits were observed in PC mode, the next 5 in WT mode, and for subsequent orbits the mode is indicated by symbols. The count rates in PC mode are corrected for the loss of effective area due to pile up with the xRTLCCORR tool. (Bottom) Magnitude of UVOT detections in the indicated filters.

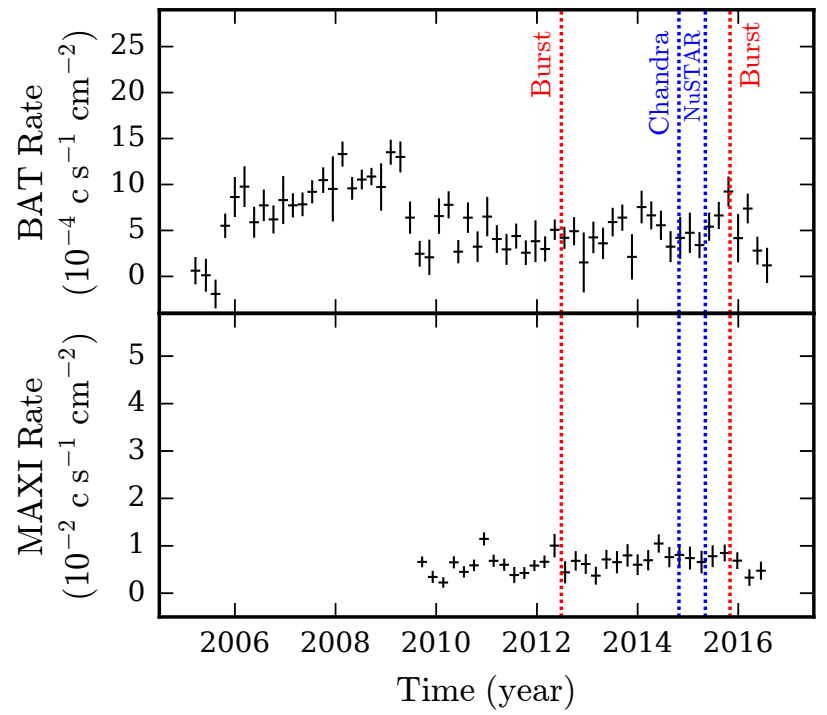

FIG. 2.- Light curve of the persistent emission from IGR J17062-6143 since its discovery in 2006. The panels show the photon flux as observed with Swift/BAT (top; $15-150 \mathrm{keV}$ band pass) and $M A X I /$ GSC (bottom; $2-10 \mathrm{keV}$ band pass) at a 70 day time resolution. Dotted lines indicate the times of the Chandra and NuSTAR observations as well as the two burst observations, where the last burst is studied in this paper. In Section 3 we study the combined MAXI spectrum accumulated between the two bursts.

$22 \%$ for $M A X I$. The corresponding hardness ratio from the $4-20 \mathrm{keV}$ and $2-4 \mathrm{keV}$ MAXI bands does not exhibit significant variability. We take this as an indication that the spectral shape did not change substantially, and we extract one $M A X I /$ GSC spectrum from all observations in that interval combined (Table 2). We restrict the energy range to $2-10 \mathrm{keV}$ where the source is detected most clearly, collecting a total of $5.5 \times 10^{3}$ net source counts. In the same period, the source was also observed with the following instruments.
The Chandra X-ray Observatory Weisskopf et al. 2000) was launched in 1999. Chandra observed the source on $10 / 25 / 2014$ and $10 / 27 / 2014$ for a total of $93 \mathrm{ks}$ with the High Energy Transmission Grating Spectrometer (HETGS; Canizares et al. 2005), which includes the High Energy Grating (HEG) and the Medium Energy Grating (MEG). We use the spectra and response matrices provided in the Chandra Grating-Data Archive and Catalog (TGCat; Huenemoerder et al. 2011). The data products were extracted using a narrow mask for better flux correction of the HEG below $6.9 \mathrm{keV}$. The background was extracted from an off-source position. We will analyze the spectra in the $0.5-10 \mathrm{keV}$ energy range of the $+1,-1$ orders of the MEG and HEG for both pointings, which have a total of $1.8 \times 10^{5}$ counts $(91 \%$ of the counts in all orders).

The Nuclear Spectroscopic Telescope Array (NuSTAR; Harrison et al. 2013) observes the hard X-ray sky since 2012 with its focusing optics and two imaging FocalPlane Modules: FPMA and FPMB. NuSTAR observed IGR J17062-6143 on 05/06/2015. The data is reprocessed with NUPIPELINE version 0.4.5 using calibration data with the time stamp $07 / 31 / 2016$, creating spectra for both modules in the $3-50 \mathrm{keV}$ band. The combined FPMA and FPMB spectra have $1.5 \times 10^{5}$ counts. Background spectra are extracted from an off-source position.

\subsection{Interstellar Absorption}

As part of our spectral model, interstellar absorption is described by the Tübingen-Boulder model with abundances from Wilms et al. (2000). The tool NHтот6 calculates the Galactic absorption column of atomic (Kalberla et al. 2005) and molecular (Schlegel et al. 1998) hydrogen using radio and infra-red maps, respectively (Willingale et al. 2013). In the direction of

${ }^{6}$ See http://www.swift.ac.uk/analysis/nhtot/ 


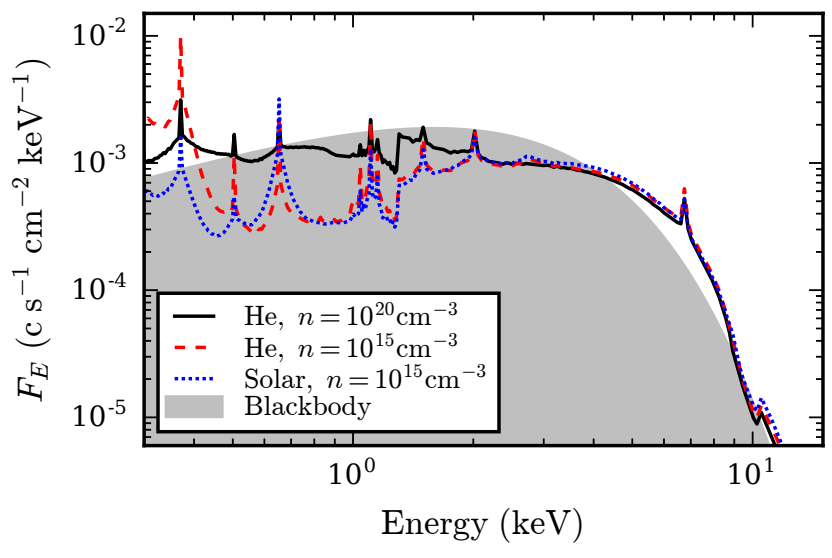

FIg. 3.- Comparison of the photon flux, $F_{E}$, as a function of energy predicted by a blackbody with temperature $k T=1.0 \mathrm{keV}$ (top of the shaded region) and three reflection models. The reflection models reprocess the blackbody for a disk with $\log \xi=3.0$, either solar composition or helium with solar metal abundance, and given number density $n$. All models have been normalized such that the integrated $E F_{E}$ is unity.

IGR J17062-6143, within $1^{\circ}$ from the source, we find a mean value of $N_{\mathrm{H}}=1.58 \times 10^{21} \mathrm{~cm}^{-2}$.

For bright low-mass X-ray binaries (LMXBs), absorption lines and edges have been used to quantify the interstellar absorption column (e.g., Pinto et al. 2010). A study of the Chandra gratings spectra of the persistent emission did not find significant absorption features that could be used for this purpose (Degenaar et al. 2016). Alternatively, $N_{\mathrm{H}}$ may be determined as part of the fit to the continuum spectrum. Indeed, $N_{\mathrm{H}}$ measured from XRT spectra of the 2012 burst from IGR J17062-6143 is consistent with NHTOT (Degenaar et al. 2013). The best-fitting value is, however, dependent on the model for the continuum spectrum, because a model that adds more flux at low energies $(E \lesssim 2 \mathrm{keV})$ requires a larger $N_{\mathrm{H}}$ to compensate (see, e.g., the fits by Degenaar et al. 2016). Because $N_{\mathrm{H}}$ cannot be robustly constrained by the X-ray spectra, we fix $N_{\mathrm{H}}$ to the value from NHтот.

\subsection{Models of Photoionized Reflection}

We employ models of reflection off a photoionized accretion disk that is illuminated by either a power law or a blackbody spectrum. Both models implement the disk as a slab of constant number density, $n$, and with ionization parameter $\xi \equiv 4 \pi F / n$, where $F$ is the irradiating flux. We will report $\log \xi$, with $\xi$ in units of $\operatorname{erg~s}^{-1} \mathrm{~cm}$.

Models of a reflected blackbody were calculated by Ballantyne (2004) for the UCXB 4U 1820-30. Because we suspect IGR J17062-6143 to be of a similar nature, the assumed composition of helium and solar metallicity is also applicable here. A density of $n=10^{15} \mathrm{~cm}^{-3}$ was assumed, although $n=10^{20} \mathrm{~cm}^{-3}$ may be more realistic for the inner disk. The density dependence is most pronounced for $E \lesssim 3 \mathrm{keV}$ (Figure 3, Ballantyne 2004). It is unimportant when fitting, e.g., $R X T E / \mathrm{PCA}$ data (Ballantyne \& Strohmaver 2004; Keek et al. 2014b) or MAXI data, and we use the Ballantyne (2004) models for the latter. For the XRT spectra, however, we calculate a new grid of models with $n=10^{20} \mathrm{~cm}^{-3}$, using the same procedure as Ballantyne (2004). The new grid covers a range of blackbody temperatures $0.2 \mathrm{keV} \leq k T \leq$ $1.2 \mathrm{keV}$ as well as $1.5 \leq \log \xi \leq 3.0$.
For reflection of a power law we employ version $0.4 \mathrm{c}$ of the relxill model (García et al. 2014; Dauser et al. 2014). relxill provides the illuminating power law with photon index $\Gamma$ and a high energy cutoff as well as the xillver model of reflection off a photoionized accretion disk (García et al. 2013). The flux ratio of the reflection and illumination components is given by the reflection fraction, $f_{\text {refl }}$ xillver assumes a density of $n=10^{15} \mathrm{~cm}^{-3}$ and a composition based on solar with a variable iron abundance. Unfortunately, the composition and density do not match the values that we preferred for blackbody reflection. Therefore, we only apply this model to $E>3 \mathrm{keV}$, where the effect of these parameters is minimal (see Figure 3 for an illustration using blackbody reflection).

relxill further takes into account relativistic effects that smooth the reflection spectrum using the relline code (Dauser et al. 2010), depending on the inclination angle of the disk with respect to the line of sight and the emissivity profile of the disk. We apply the same smoothing to the blackbody reflection models using the relconv convolution model, which is also based on the relline code.

\section{ANALYSIS OF PERSISTENT EMISSION}

We analyze the persistent spectra with XSPEC v.12.9.0i (Arnaud 1996). For all spectra, neighboring spectral bins that have fewer than 15 counts are grouped to ensure that the uncertainties in the data points are close to Gaussian. We use $\chi^{2}$ statistics and report the $1 \sigma$ uncertainties in the fit parameters. We first employ a phenomenological model, and next interpret the spectra with models that include Comptonization and reflection.

\subsection{Phenomenological fit}

To quantify the persistent emission between the 2012 and 2015 bursts, we analyze the MAXI, Chandra, and $N u S T A R$ spectra listed in Table 2. Because the flux exhibits only minor variability in that period (Figure2), we assume that the spectral shape was unchanged. All spectra are fit jointly, allowing for multiplication factors between MAXI, NuSTAR FPMA, NuSTAR FPMB, Chandra observation 15749, and Chandra observation 17543 (using XSPEC model constant). The factor for the latter is fixed to unity. We first fit a model that includes a blackbody with temperature $k T$ and normalization $K_{\mathrm{bb}}$, as well as a power law with photon index $\Gamma$. Instead of the power law normalization, we report the $0.5-10 \mathrm{keV}$ unabsorbed flux of the power law provided by a cflux component in XSPEC. Interstellar absorption is implemented as described in Section 2.3. The complete XSPEC model is constant*TBabs (bbodyrad + cflux*powerlaw). It provides a reasonable fit to the data (Figure 4 top). The residuals of the Chandra/MEG data show some features near $1 \mathrm{keV}$, but the HEG is less sensitive at those energies and its spectra does not exhibit them. The residuals for the NuSTAR data show an emission line near $6.4 \mathrm{keV}$ and a broad excess at $E>20 \mathrm{keV}$, which may be the $\mathrm{Fe} \mathrm{K} \alpha$ line complex and the Compton hump, respectively (2nd panel of Figure 4), that are known to be produced by photoionized reflection. For $N u S T A R$ we exclude the parts of the spectra where reflection features appear: $6.0 \mathrm{keV}<E<7.5 \mathrm{keV}$ and 


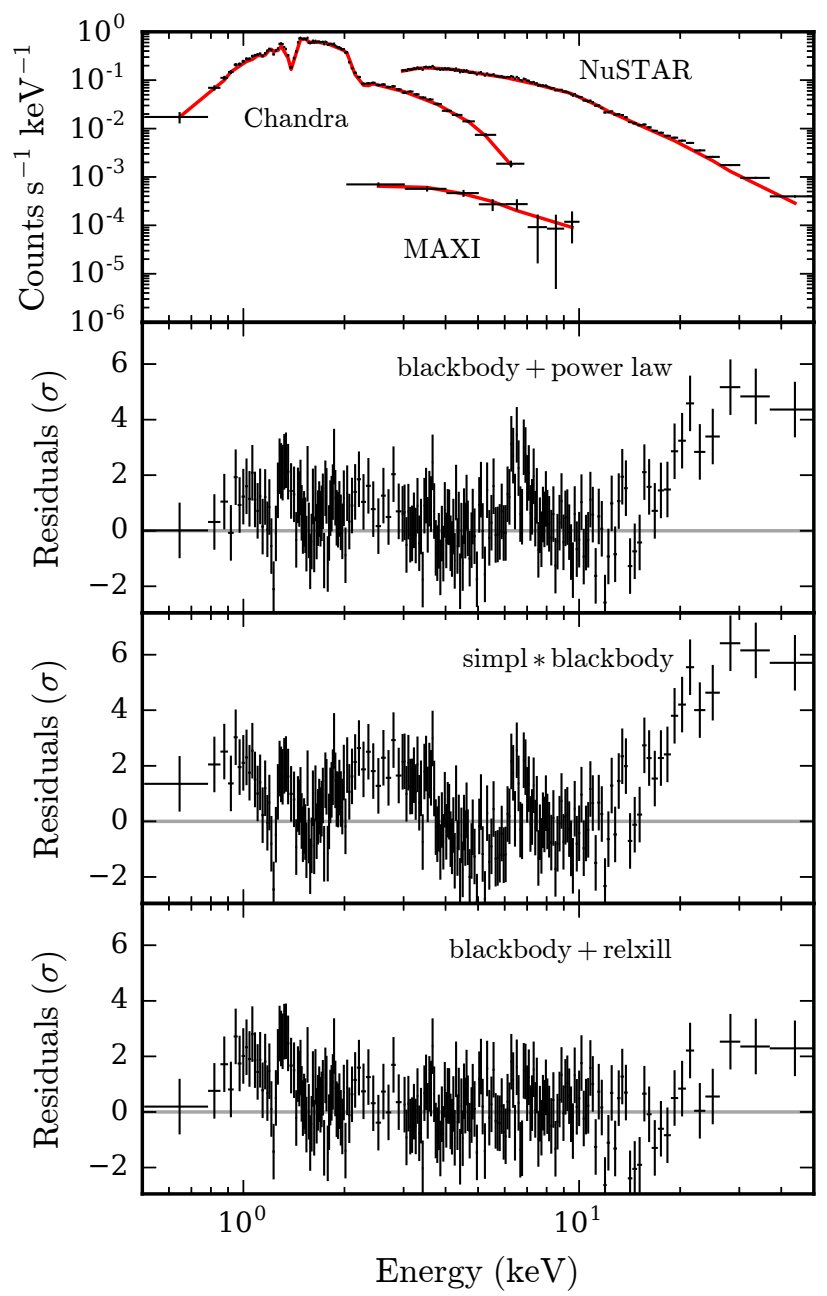

FIG. 4. - The persistent spectrum (top panel) and residuals of fits with the indicated models (other panels). We show the spectra of MEG order 1 from Chandra pointing 17543, NuSTAR FPMA, and $M A X I / G S C$, and the spectra have been rebinned for clarity. The residuals include the MEG up to $3 \mathrm{keV}$ and the FPMA in its full $3-50 \mathrm{keV}$ band. The solid line in the top panel indicates the fit with the blackbody + power law model, whose residuals in the 2nd panel exhibit an Fe $\mathrm{K} \alpha$ line near $6.4 \mathrm{keV}$ and a Compton hump above $20 \mathrm{keV}$. Those features are largely described by the relxill component of photoionized reflection (bottom panel).

$20.0 \mathrm{keV}<E<50 \mathrm{keV}$. In principle the reprocessing of the spectrum by reflection can also influence the measured continuum parameters such as $\Gamma$, but we find this not to be important effect in this case (see also the discussion in Keek \& Ballantyne 2016). The best-fitting parameter values are presented in Table 3 . The scaling factor for Chandra pointing 15749 is consistent with the ratio of the count rates in the two Chandra pointings of 0.981 . The ratio of the factors for NuSTAR FPMA and FPMB is $1.02 \pm 0.02$ : the two spectra are consistent. The scaling factor for MAXI indicates that the long-term average flux was a bit lower than at the times of the Chandra and NuSTAR pointings. Furthermore, the spectrum is dominated by the power law: the ratio of the $0.5-10 \mathrm{keV}$ power law flux and the bolometric blackbody flux is $4.6 \pm 0.3$.
TABLE 3

Fit of the Persistent Spectra with a Blackbody and POWER LAW

\begin{tabular}{cc}
\hline bbodyrad & \\
$k T(\mathrm{keV})$ & $0.456 \pm 0.005$ \\
$K_{\mathrm{bb}}(\mathrm{km} / 10 \mathrm{kpc})^{2}$ & $48 \pm 3$ \\
\hline powerlaw & $2.159 \pm 0.008$ \\
$\Gamma$ & $1.03 \pm 0.02$ \\
$F_{\mathrm{po}}\left(10^{-10} \mathrm{erg} \mathrm{s}^{-1} \mathrm{~cm}^{-2}\right)^{a}$ & scaling relative to Chandra 17543 \\
constant & $0.974 \pm 0.007$ \\
Chandra 15749 & $0.923 \pm 0.010$ \\
$N u S T A R /$ FPMA & $0.907 \pm 0.010$ \\
$N u S T A R /$ FPMB & $0.54 \pm 0.04$ \\
$M A X I$ & $0.58(9455)$ \\
\hline$\chi_{\nu}^{2}$ (degrees of freedom) & \\
\hline Unabsorbed flux in the $0.5-10 \mathrm{keV}$ band for Chandra ObsID \\
17543
\end{tabular}

TABLE 4

Fit of the Persistent Spectra with a Blackbody and SIMPL

\begin{tabular}{cc}
\hline bbodyrad & $0.298 \pm 0.006$ \\
$k T(\mathrm{keV})$ & \\
\hline simpl & $2.250 \pm 0.007$ \\
$\Gamma$ & $0.664 \pm 0.013$ \\
\hline$f_{\mathrm{sc}}$ & $0.973 \pm 0.007$ \\
constant & $0.882 \pm 0.010$ \\
Chandra 15749 & $0.865 \pm 0.010$ \\
$N u S T A R$ FPMA & $0.54 \pm 0.04$ \\
$N u S T A R$ FPMB & $1.70 \pm 0.02$ \\
$M A X I$ & $0.62(9455)$ \\
\hline$F_{\text {bol }}\left(10^{-10} \mathrm{erg} \mathrm{s}^{-1} \mathrm{~cm}^{-2}\right)^{a}$ & scaling relative to Chandra \\
$\chi_{\nu}^{2}($ degrees of freedom $)$ & \\
\hline
\end{tabular}

${ }^{a}$ Unabsorbed bolometric flux in the $0.01-100 \mathrm{keV}$ band for Chandra spectrum 17543

\subsection{Simpl Comptonization Model}

To measure the bolometric flux, the model needs to be extrapolated outside of the combined instrument bands. The power law component poses a problem, as it strongly increases towards lower energies. A power law component from accreting compact objects is often explained as the result of Inverse Compton scattering in an accretion disk corona. In our case, flux from the blackbody component could be Comptonized by hot electrons in a corona. In the spectral model we replace the power law by the simpl model (Steiner et al. 2009) convolved with the blackbody. This is an empirical Comptonization model that takes a "scattering fraction", $f_{\mathrm{sc}}$, of the blackbody flux, and produces a power law with photon index $\Gamma$ towards higher energies from Compton upscattering. Towards lower energies, the downscattered flux falls off quickly with energy. A cflux component is used to measure the total unabsorbed bolometric flux in the $0.01-100 \mathrm{keV}$ range. The complete XSPEC model becomes constant*TBabs*cflux*simpl*bbodyrad. The quality of the fit is similar to the previous fit (Table 4). The largest difference is a lower $k T$ : simpl rolls off at the lower energies, and the blackbody moves to lower energies to compensate. In turn, the flux is now underpredicted around $\sim 3 \mathrm{keV}$ (3rd panel of Figure 4). If Comptonization of the blackbody is the correct interpretation of the power law at higher energies, the excess at lower energies must be produced by another process. Photoionized reflection off the accretion disk could pro- 
TABLE 5

Fit of the NuStaR Persistent Spectra with a Blackbody and a Reflected Power Law.

\begin{tabular}{cc}
\hline bbodyrad & $0.48 \pm 0.02$ \\
$k T(\mathrm{keV})$ & $44 \pm 14$ \\
$K_{\mathrm{bb}}(\mathrm{km} / 10 \mathrm{kpc})^{2}$ & $2.060 \pm 0.013$ \\
\hline relxill & $3.28 \pm 0.08$ \\
$\Gamma$ & $0.31 \pm 0.04$ \\
$\log \xi$ & $2.1_{-1.3}^{+1.9 p}$ \\
$f_{\text {reff }}$ & $0.716 \pm 0.004$ \\
$R_{\text {in }}\left(10^{2} R_{\mathrm{g}}\right)$ & scaling relative to FPMA \\
$F_{3-50 \mathrm{keV}}\left(10^{-10} \mathrm{erg} \mathrm{s}^{-1} \mathrm{~cm}^{-2}\right)^{a}$ & $0.980 \pm 0.006$ \\
\hline constant & $0.97(974)$ \\
\hline NuSTAR FPMB &
\end{tabular}

$a \frac{\chi_{\nu}}{\text { Unabsorbed bolometric flux in the } 3.0-50 \mathrm{keV} \mathrm{NuSTAR} \text { band }}$ of the relxill component of the FPMA spectrum

duce this in combination with the $\mathrm{Fe} \mathrm{K} \alpha$ line near $6.4 \mathrm{keV}$ and the Compton hump.

\subsection{Relxill Reflection Model}

We test the reflection interpretation using the relxill model (Section 2.4), with the complete XSPEC model being constant*TBabs (bbodyrad + cflux*relxill). It is a complex model that, when fit to data of modest quality, presents multiple degenerate solutions. For example, there are 8 parameters that shape the Fe $\mathrm{K} \alpha$ line. Moreover, several potentially important effects are not taken into account, such as the dependence on the density of the disk and the low-energy turn-off of the illuminating power law (fixed at $0.1 \mathrm{keV}$; García et al. 2013). In fitting this model, one runs the risk of certain parameters taking on unphysical values in order to compensate for these deficiencies. Indeed, when left unconstrained, the fit prefers a maximally spinning neutron star $(a=1)$ and an iron abundance of the disk of 10 times solar (the maximal value provided by the model; see also Degenaar et al. 2016). Therefore, we fix several parameters to reasonable values. We assume a solar iron abundance. The disk emissivity is taken to decrease with the 3rd power of the radius, and the disk's outer radius is fixed at a large value of $400 R_{\mathrm{g}}\left(R_{\mathrm{g}}=G M / c^{2}\right.$ is the gravitational radius). As no eclipses or dipping are apparent in the light curves, the disk's inclination is likely less than $\sim 60^{\circ}$ : we choose a value of $30^{\circ}$. Similarly, the fastest spinning neutron star known in an LMXB has spin $a \simeq 0.3$ (e.g., Degenaar et al. 2015): we choose a value of $a=0.15$. During the fits, the high energy cutoff pegs at the domain boundary of $1000 \mathrm{keV}$. Therefore, we fix the cutoff energy to this value. Furthermore, the mentioned dependencies of the reflection spectrum on density and low-energy turn-off are strongest at $E \lesssim 3 \mathrm{keV}$ (Section 2.4). We, therefore, limit the fit to the NuSTAR spectra in their full $3.0-50.0 \mathrm{keV}$ band.

We perform a fit with these constraints (Table [5), which is largely consistent with the results from Degenaar et al. (2016). The blackbody parameters are consistent within $1 \sigma$ with the fit of the blackbody + power law model (Table 3), whereas the power law's $\Gamma$ is $5 \%$ smaller. The inner disk radius is large: $R_{\text {in }}=210 R_{\mathrm{g}}$. The width of the Fe $\mathrm{K} \alpha$ line is influenced by $R_{\text {in }}$ as well as the neutron star spin and the disk's inclination. Repeating the fit for spin $0<a<0.3$ and for inclination angles up to $60^{\circ}$ always leads to similarly large $R_{\text {in }} \gtrsim 10^{2} R_{\mathrm{g}}$.
A local minimum in $\chi^{2}$ is present at $\log \xi \simeq 1.6$ and a global minimum at $\log \xi=3.28 \pm 0.08$. The latter indicates that the reflecting material is highly ionized. The reflection model provides a good description of the $\mathrm{Fe} \mathrm{K} \alpha$ line (Figure 4 bottom), but a minor part of the Compton hump remains visible in the residuals; its precise peak energy depends on the density of the reflector (García et al. 2016). Although we only fit to the $\mathrm{NuS}$ $T A R$ spectra, we also show the residuals of a Chandra spectrum with respect to the best-fitting model (Figure 4 bottom). The features near $1 \mathrm{keV}$ remain visible. This part of the spectrum is most sensitive to density, or alternatively these features could be produced by a local warm absorber. We refer to Degenaar et al. (2016) for an in-depth discussion of the line features.

\section{ANALYSIS OF BURST EMISSION}

The X-ray count rates of the burst observations (Figure 1 top) exhibit a power law decline in the initial $1.6 \times 10^{5} \mathrm{~s}$ since the $M A X I$ trigger (allowing for a small offset between the PC and WT mode due to the accuracy of the pile-up correction and differences in the grade selection). In contrast to the burst from 2012 (Degenaar et al. 2013), no strong variability is visible on short time scales, although this may have been missed due to the sparse sampling in the first few hours. After this period (2 days since the trigger), the count rate drops sharply. Over the subsequent 1.4 weeks, the count rate slowly increases to a value of $\sim 1.2 \mathrm{cs}^{-1}$. The magnitudes of the UVOT detections exhibit similar behavior (Figure 1 bottom), suggesting that both parts of the spectrum are powered by the same emission source, either directly or after reprocessing. Considering thermal emission from a neutron star undergoing a thermonuclear burst, a blackbody that peaks in the X-rays at $1 \mathrm{keV}$ would produce a magnitude difference of $\Delta M \simeq 1.2$ between the UVW2 and U filters. No such offset is apparent between the magnitudes of different filters, which indicates that the spectrum in the UV regime is relatively flat, possibly due to reprocessing of the burst emission by the disk (e.g., Ballantyne 2004).

Similar to the analysis of the persistent emission, we fit the burst spectra both with a phenomenological model and a reflection model.

\subsection{Phenomenological Spectral Fits}

We illustrate our choice of spectral model using the first XRT spectrum (Figure [5). The spectrum is dominated by a blackbody (Negoro et al. 2015; Iwakiri et al. 2015), but excesses are visible at both low and high energy (2nd panel of Figure 5). Adding a power law provides a reasonable description, although some structure remains in the residuals (3rd panel of Figure 55). The strongest is an emission feature around $1 \mathrm{keV}$ (see also Degenaar et al. 2013) which can be fit with a Gaussian profile (4th panel of Figure 5). The complete model is, therefore, similar to the persistent model in Section 3.1 with the addition of the Gaussian: the TBabs (bbodyrad + cflux*powerlaw + gaussian), where interstellar absorption is again implemented with a fixed $N_{\mathrm{H}}$ (Section 2.3).

This model is fit to all XRT spectra. The $M A X I$ spectra, however, do not cover the $1 \mathrm{keV}$ line and do not 


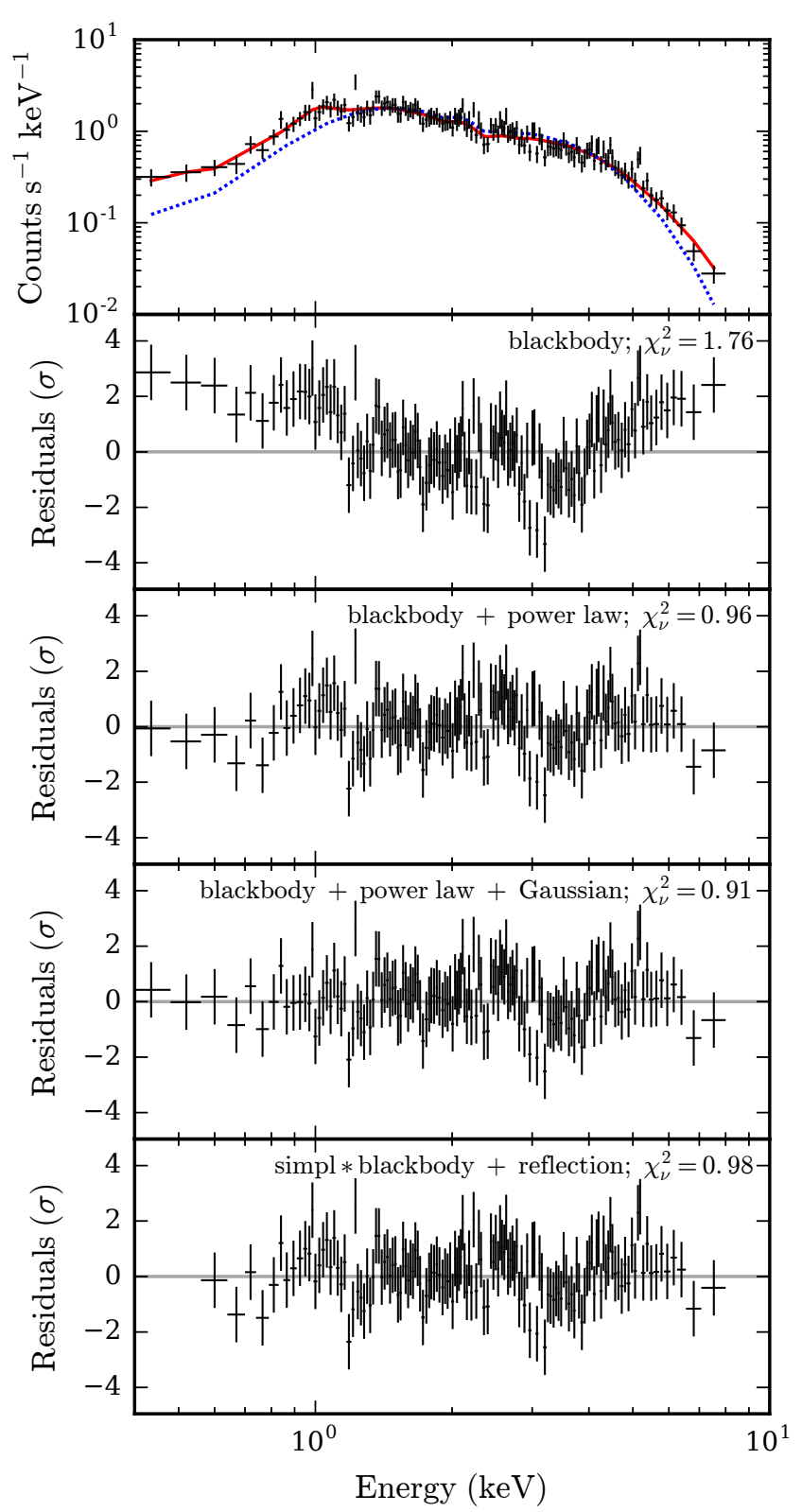

FIG. 5.- (Top) First XRT burst spectrum as a function of energy. The dotted line is the best fit with an absorbed blackbody model and the solid line results from a model that further includes a power law and a Gaussian line (see also the second and fourth panels, respectively). (Below) Residuals of spectral fits with indicated models and goodness of fit; in units of the $1 \sigma$ uncertainty of the data points. A blackbody describes most of the data, whereas a power law can fit the excesses at low and high energy. The most prominent remaining feature in the residuals can be well fit with a Gaussian emission line at $\sim 1.0 \mathrm{keV}$.

require the power law. Those two spectra are fit with only an absorbed blackbody (Figure 6).

The blackbody temperature decreases from $k T=$ $1.47 \pm 0.05 \mathrm{keV}$ in the first $(M A X I)$ data point to a mean value of $k T=0.291 \pm 0.005 \mathrm{keV}$ at the end in a week of XRT pointings (Figure 7 ). The first data point indicates photospheric radius expansion (PRE), as the blackbody normalization is an order of magnitude larger than the mean value for subsequent spectra. We calculate the

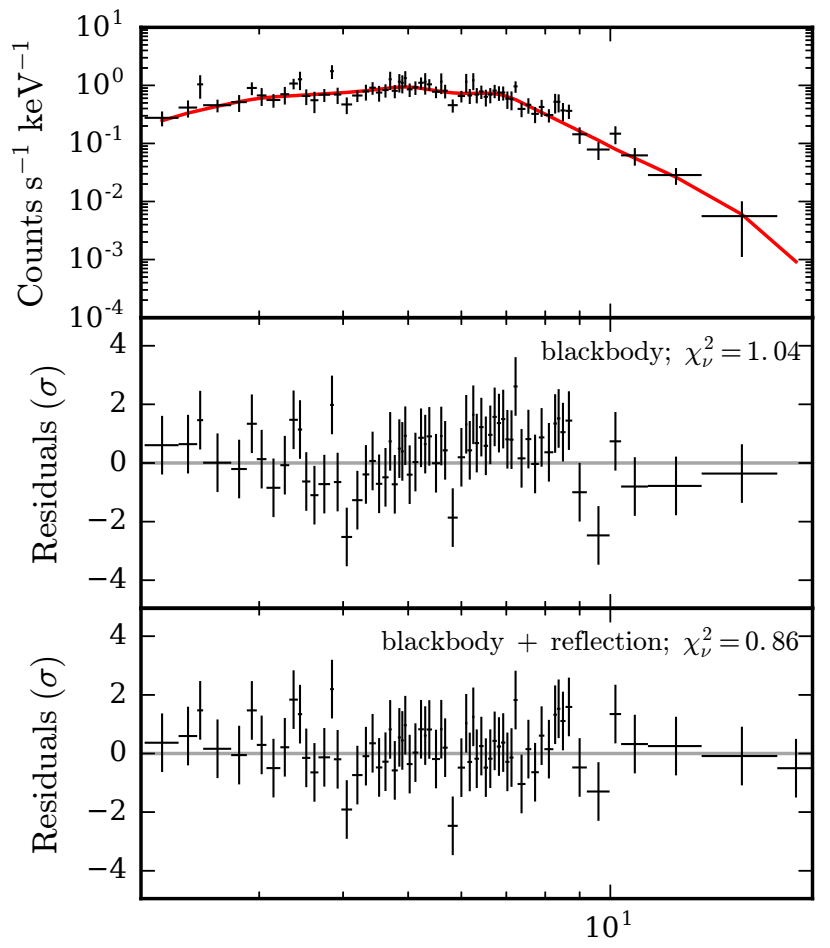

Energy (keV)

Fig. 6.- Similar to Figure 5 for the first $M A X I /$ GSC burst spectrum. The solid line in the top panel corresponds to the best fitting blackbody model that includes a reflection component (see also the bottom panel). After a fit with an absorbed blackbody, a broad excess around $\sim 6.5 \mathrm{keV}$ and a deficit above $\sim 9 \mathrm{keV}$ remain (middle panel). These features are well described with a model of photoionized reflection (bottom).

bolometric unabsorbed blackbody flux (Figure 8 top).

The power law index evolves over time: the first 6 XRT spectra yield a weighted mean of $\Gamma=1.31 \pm 0.05$, and we find $\Gamma=1.72 \pm 0.06$ for the last 8 spectra. In between, $\Gamma$ displays some variability. We determine the unabsorbed in-band $(0.5-10 \mathrm{keV})$ power law flux, $F_{\mathrm{po}}$, and take the ratio to the blackbody flux (on average $95 \%$ of the bolometric blackbody flux falls in the $0.5-10 \mathrm{keV}$ band; Figure 8 bottom). In the first 6 XRT spectra the weighted mean is $0.66 \pm 0.04$. The ratio increases over time: for the last 8 spectra it is $1.01 \pm 0.09$.

The Gaussian emission feature near $1 \mathrm{keV}$ is outside of the MAXI/GSC band, but it is a significant component for the first 7 XRT spectra. The weighted mean of the centroid energy is $1.035 \pm 0.009 \mathrm{keV}$. The width of the Gaussian is small: typically $\sim 10^{-2} \mathrm{keV}$, which is consistent with being unresolved. The normalization, $K_{\text {Gauss }}$, decreases at the same rate as the blackbody flux.

\subsection{Reflection Fits}

The phenomenological fits suggest that the burst flux, as represented by the blackbody that dominates the spectrum, is reprocessed into the power law and Gaussian components, whose fluxes follow the blackbody flux. The flux fraction (Figure 8) and the power law's photon index (Figure 7) are different in the early and the later observations. This may indicate that two reprocessing regions are active, and their relative contributions to the flux change with time. Similar to our model of the persistent 


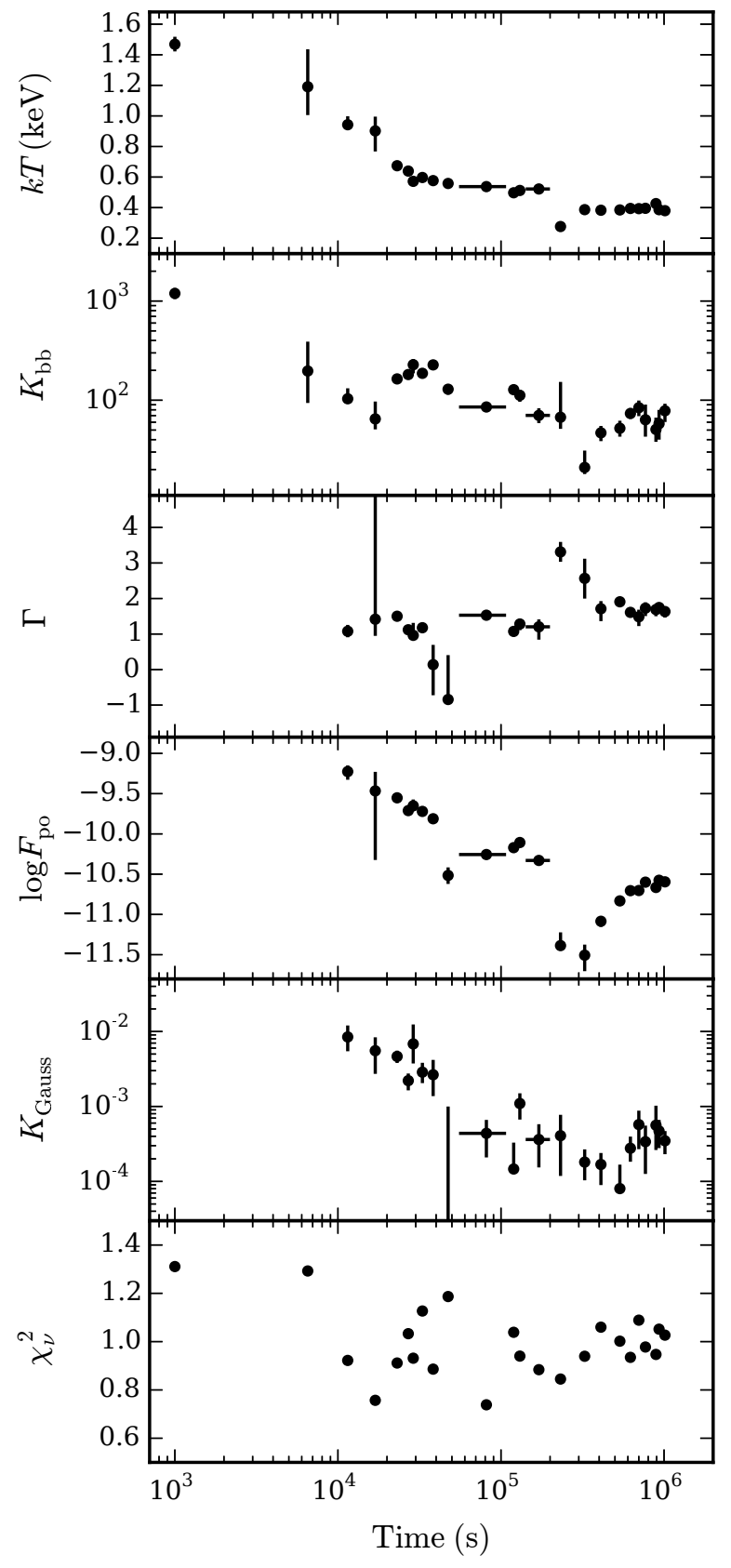

FIG. 7.- Best fit values and $1 \sigma$ uncertainties as a function of time from analysis of the $M A X I$ and XRT burst spectra. The spectral components include a blackbody (temperature $k T$ and normalization $K_{\mathrm{bb}}$ ), power law (photon index $\Gamma$ and $0.5-10 \mathrm{keV}$ unabsorbed flux $F_{\text {po }}$ ), and a narrow Gaussian at $1 \mathrm{keV}$ (normalization $\left.K_{\text {Gauss }}\right)$. The bottom panel shows the goodness of fit per degree of freedom, $\chi_{\nu}^{2}$. Horizontal "error bars" indicate the width of the time interval during which the spectrum of a data point was observed. Normalizations are in units of $\mathrm{cs}^{-1} \mathrm{~cm}^{-2} \mathrm{keV}^{-1}$. For easier viewing, we place the burst start at $10^{3} \mathrm{~s}$ before the first data point, but the time offset is most likely smaller (see Figure 8).

emission, we replace the power law by a simpl component for Comptonization of the blackbody emission (Section 3.2) and a reflection component. Because the burst emission is dominated by the blackbody, we use a model of a reflected blackbody, which has the same temperature as the blackbody component (2.4). The full model is:

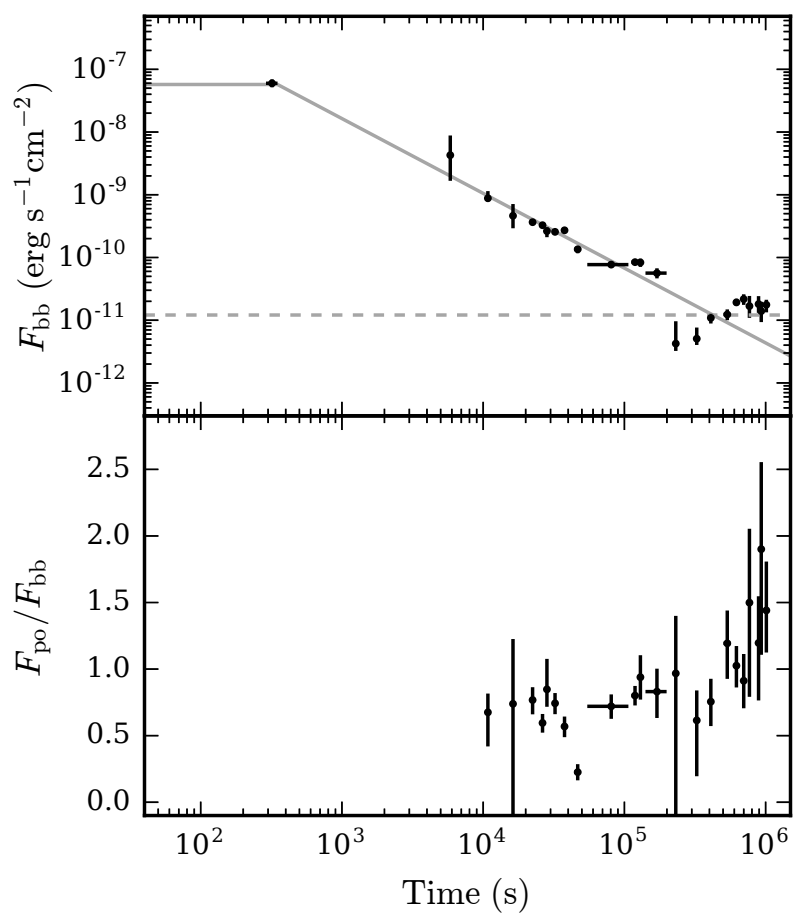

Fig. 8.- (Top) Unabsorbed bolometric flux from the blackbody component, $F_{\mathrm{b}}$, as a function of time since $320 \mathrm{~s}$ before the first data point. The solid line indicates the best-fit model of the flux profile (Equation 1) to the first $4 \times 10^{4} \mathrm{~s}$, and the dashed line indicates the pre-burst blackbody flux (Table 3). (Bottom) The ratio of the $0.5-10 \mathrm{keV}$ power law flux, $F_{\mathrm{po}}$ (Figure 7), and $F_{\mathrm{bb}}$. For the first two data points $F_{\text {po }}$ is not measured.

TBabs (simpl*bbodyrad+cflux*relconv*reflection).

After we replace the power law with these two components, competition between them during the fit yields large uncertainties in the model parameters. We, therefore, fit all burst spectra simultaneously and constrain certain parameters to be the same for each spectrum, similar to the procedure followed by, e.g., Keek et al. $(2014 \mathrm{~b})$. Specifically, each spectrum has its own value of the blackbody parameters $k T$ and $K_{\mathrm{bb}}$ and the unabsorbed bolometric reflection flux $F_{\text {refl }}$ (determined in the $0.01-100 \mathrm{keV}$ band), whereas the spectra share the ionization parameter $\log \xi$, the inner radius of the reflection site $R_{\text {in }}$, as well as $\Gamma$ and $f_{\text {sc }}$ of the simpl component. Parameters of the relconv component (other than $R_{\text {in }}$ ) are the same as for the persistent emission (Section 3.3).

We fit the model to the XRT spectra, and find that after the first 6 spectra, the reflection component can no longer be distinguished. Therefore, we perform the simultaneous fit to the first 6 XRT spectra, which cover the initial 9 hours of the burst. We obtain a good fit with $\chi_{\nu}^{2}=1.04$ (Table 6] and Figure 9). The fit residuals are similar to those for the phenomenological model (Figure 6 bottom).

Of the MAXI spectra, only the first has sufficient counts to distinguish deviations from a pure blackbody. No power law component was found with the phenomenological spectral model (Figure 6 2nd panel), and a fit with the reflection model finds a vanishingly small $f_{\text {sc }}$. Therefore, we fit the first $M A X I$ spectrum without the simpl component (Table 6 and Figure 9). The reflection component successfully describes the features in the 
TABLE 6

Fit of the Burst Spectra with a Comptonized Blackbody and Reflection. ${ }^{a}$

\begin{tabular}{ccc}
\hline & $M A X I / \mathrm{GSC}$ & Swift $/ \mathrm{XRT}$ \\
\hline $\begin{array}{c}\text { blackbody reflection } \\
\log \xi\end{array}$ & $2.7_{-0.2}^{+0.4}$ & $3.0_{-0.2}^{+0 \mathrm{p}}$ \\
\hline relconv & $\left(4.0_{-3.0}^{+0 \mathrm{p}}\right) \times 10^{2}$ & $14_{-7}^{+25}$ \\
$R_{\text {in }}\left(R_{\mathrm{g}}\right)$ & - & \\
simpl & - & $2.3_{-0.3}^{+0.2}$ \\
$\Gamma$ & - & $0.56_{-0.10}^{+0.12}$ \\
$f_{\mathrm{sc}}$ & $0.86(61)$ & $1.04(1296)$
\end{tabular}

${ }^{a}$ A subsection of the fit parameters is listed here for the first $M A X I$ scan and the first 6 XRT spectra, where reflection is detected. The XRT spectra are fit simultaneously, and the listed values are shared between the spectra. The fit parameters that are allowed to vary for each spectrum are shown in Figure 9

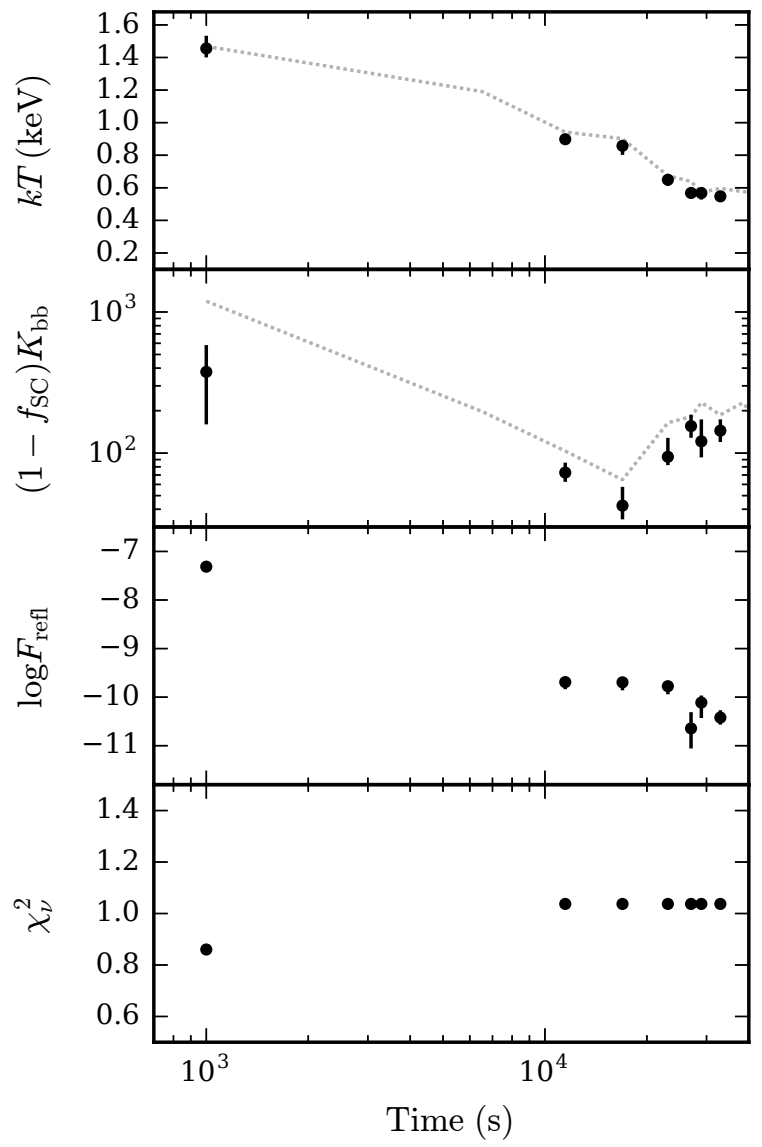

FIG. 9.- Similar to Figure 7 for the spectral fits with a reflection model. The spectral components include a blackbody (temperature $k T$ and normalization $K_{\mathrm{bb}}$ ) and a reflected blackbody (disk ionization parameter $\log \xi$ and unabsorbed bolometric flux $\left.F_{\text {refl }}\right)$. Part $\left(f_{\mathrm{sc}}\right)$ of the blackbody is Comptonized, such that the normalization of the pure blackbody is $\left(1-f_{\mathrm{sc}}\right) K_{\mathrm{bb}}$. Dotted lines indicate values from the blackbody fit (Figure 7), and arrows designate lower limits.

residuals of the phenomenological fit (Figure 6 bottom).

Compared to the phenomenological fits, $k T$ is on average $6 \%$ smaller. $K_{\mathrm{bb}}$ is $55 \%$ larger, but part of the blackbody flux is Comptonized by simpl. The pure blackbody normalization is $\left(1-f_{\mathrm{sc}}\right) K_{\mathrm{bb}}$, which is $32 \%$ smaller than the phenomenological $K_{\mathrm{bb}}$. The changes in $k T$ and $K_{\mathrm{bb}}$ partially compensate each other, such that the black-

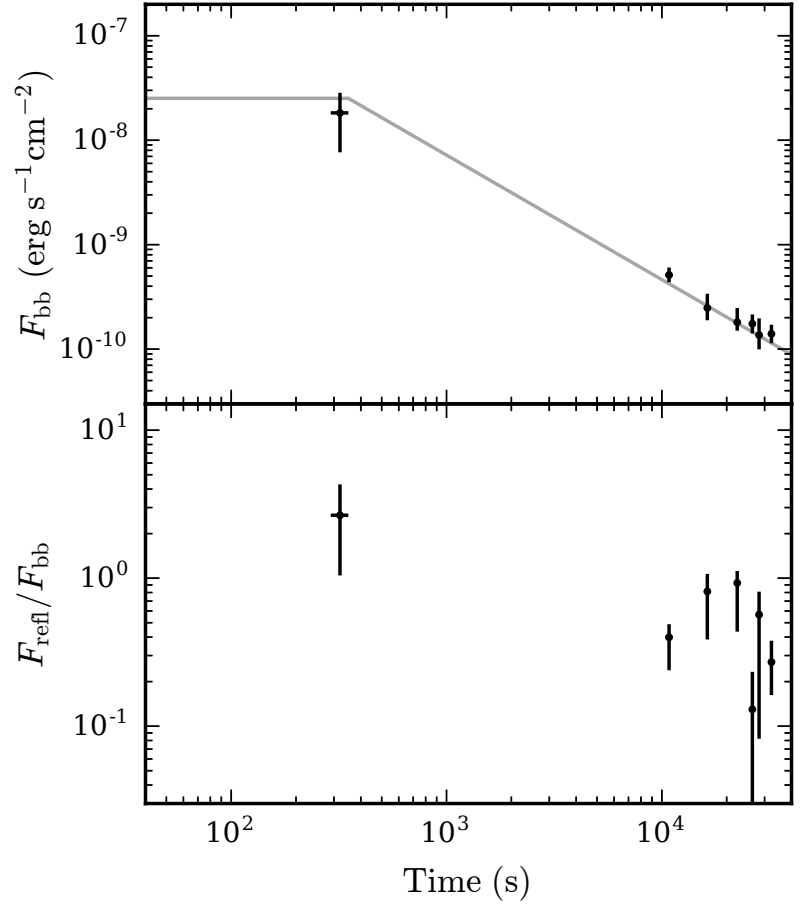

Fig. 10.- Similar to Figure 8 for the spectral fits with a reflection model (Figure 9). The bottom panel shows the ratio of the unabsorbed bolometric fluxes of the reflection $\left(F_{\text {refl }}\right)$ to the blackbody $\left(F_{\mathrm{bb}}\right)$ component. The solid line is the burst profile fit to the results from the phenomenological fit multiplied by $\left(1-f_{\mathrm{sc}}\right)$ (Figure 8)

body flux is lower by a factor $\left(1-f_{\mathrm{sc}}\right)$ (Figure [10). The reflection fraction, $F_{\text {refl }} / F_{\text {bb }}$, has a weighted mean of $0.39 \pm 0.05$ for the XRT spectra. Interestingly, the flux of the first $M A X I$ spectrum is also consistent with being lower by a factor $\left(1-f_{\mathrm{sc}}\right)$. Its reflection fraction may, however, be substantially larger: $F_{\text {refl }} / F_{\text {bb }}=3 \pm 2$.

\subsection{Burst Properties}

To determine the properties of the burst, we consider the first $10.5 \mathrm{hr}$, because for later spectra the power law flux does not show smooth behavior (Figure 7), indicating that other effects besides cooling start playing a role. This time interval includes the $2 M A X I$ spectra and the first 7 XRT spectra. We use the blackbody flux from the phenomenological fits to determine the burst properties (see also discussion in Section 5.5).

The unabsorbed bolometric peak blackbody flux is $(6.0 \pm 0.9) \times 10^{-8} \mathrm{erg} \mathrm{cm}^{-2} \mathrm{~s}^{-1}$ in the first $M A X I$ scan, and the blackbody normalization is substantially larger than in subsequent measurements. It is likely an episode of PRE where the Eddington limit is reached. Because of the PRE phase, we expect that the burst had a fast rise to the Eddington limit ( $\lesssim 1 \mathrm{~s}$; e.g., in't Zand et al. $2014 \mathrm{~b})$, and that the flux stayed at this value until the end of PRE at time $t_{\mathrm{PRE}}$, followed by a power law decay with index $\alpha$ produced by the cooling neutron star envelope. This leads to the following simple model of the burst flux as a function of time:

$$
\begin{aligned}
& F\left(t<t_{\mathrm{PRE}}\right)=F_{\mathrm{Edd}} \\
& F\left(t>t_{\mathrm{PRE}}\right)=F_{\mathrm{Edd}}\left(\frac{t}{t_{\mathrm{PRE}}}\right)^{-\alpha}
\end{aligned}
$$


We fit this model to the burst flux. Because the first MAXI scan exhibits PRE and the second does not, we constrain $t_{\mathrm{PRE}}$ to lie between those two. The start time of the burst is unknown. We, therefore, take a time offset, $t_{0}$, into account with respect to the center of the first $M A X I$ scan. $t_{0}=30 \mathrm{~s}$ places the burst start at the beginning of the $M A X I$ scan, and $t_{0}=92$ minutes places it at the start of the data gap preceding the scan. We perform the fit for a linear grid of 1000 values for $t_{0}$ in this range. The effect of $t_{0}$ is largest for the first data point: the fit is worse for increasing $t_{0}$, because for large $t_{0}$ the first data point lies above the power law implied by the other points. For each value of $t_{0}$ we determine the likelihood of the fit by calculating the probability of obtaining the measured $\chi^{2}$ or larger for 6 degrees of freedom. Fits with $t_{0}>6.1 \times 10^{2} \mathrm{~s}$ are disfavored at a $95 \%$ confidence level: the burst start was likely at most 10 minutes before the first $M A X I$ scan.

For each fit we determine the optimal values of the parameters and their $1 \sigma$ positive and negative errors. We represent these by distributions that peak at the optimal value and have Gaussian "wings" to lower and higher values as appropriate for the asymmetric errors. The distributions are scaled by the likelihood of each fit, and then summed for a given parameter over all fits. This produces a distribution that includes the uncertainty in $t_{0}$ as well as the likelihood of each value of $t_{0}$. It typically has a bell shape with asymmetric wings. We report the value where the distribution peaks as the optimal value, and integrate the wings outwards to locate the $68 \%$ confidence regions. We find $\alpha=1.15_{-0.12}^{+0.14}, F_{\text {Edd }}=\left(5.7_{-0.7}^{+1.0}\right) \times 10^{-8} \mathrm{erg} \mathrm{s}^{-1} \mathrm{~cm}^{-2}$, and $t_{\mathrm{PRE}}=\left(1.7_{-1.1 \mathrm{p}}^{+1.7}\right) \times 10^{2} \mathrm{~s}$, where ' $\mathrm{p}$ ' indicates for $t_{\mathrm{PRE}}$ that the lower error is pegged to the end of the first MAXI scan. $F_{\mathrm{Edd}}$ is slightly lower than the flux value of the first data point, because a lower $F_{\text {Edd }}$ is preferred for larger values of $t_{0}$. The difference is, however, only $0.3 \sigma$. For each fit we calculate the burst fluence, $\mathcal{F}_{\text {burst }}$, and the ratio of the fluence and peak flux, $\tau$, and combine the results in the same way as the fit parameters: $\mathcal{F}_{\text {burst }}=\left(7.2_{-1.3}^{+2.2}\right) \times 10^{-5} \mathrm{erg} \mathrm{cm}^{-2}$ and $\tau=\left(1.3_{-0.3}^{+0.4}\right) \times 10^{3} \mathrm{~s}$, where the fluence is calculated from $t=0$ to the end of the 9 th data point where we ended the fit.

Now that we have a description of the flux decay, we check whether the time resolution that we employ is sufficient. During the exposure interval of each spectrum, we compare the expected change in the blackbody temperature from the central value (half of the total change), $\Delta k T$, to the uncertainty, $\sigma$, in the measured $k T$ for the respective spectrum. For the majority of spectra, $\Delta k T \lesssim 1 \sigma$, and for one spectrum $\Delta k T=3.7 \sigma$. Therefore, for all but the latter case the change in $k T$ is small enough to allow for fitting with a single temperature blackbody. The worst case is the spectrum just before $t=10^{5} \mathrm{~s}$, which spans a substantially longer time interval than the other spectra. Because this spectrum was taken after the first $10.5 \mathrm{hr}$ where we determine the burst properties, and because the spectral parameters do not exhibit especially deviant behavior, we forego splitting the spectrum in shorter time intervals.

\section{DISCUSSION}

We first investigate the properties of the burst and persistent emission using the phenomenological fits. Next, we use the results of the spectral analysis to form a picture of the accretion environment in IGR J17062-6143, and discuss the implications of reflection and anisotropic emission on the burst properties.

\subsection{Burst Ignition}

Using the blackbody component from the phenomenological spectral model (Section 4.1), we determined the properties of the burst (Section 4.3). The burst fluence is $\sim 4$ times larger than what was inferred for the 2012 burst from IGR J17062-6143 (Degenaar et al. 2013). The burst is observable for hours, similar to superbursts (e.g., Keek \& in 't Zand 2008). This is in part due to the low persistent flux, which allows the burst to be detectable for many hours. The decay time scale $\tau$ of 21 minutes is, however, indicative of a deep helium flash. Nevertheless, it may be the longest helium flash observed thus far (e.g., Linares et al. 2012). For several superbursts from other sources with low mass accretion rates, there remains ambiguity on whether the fuel consists of carbon or helium (e.g., Kuulkers et al. 2010; Altamirano et al. 2012).

We regard the flux of the first MAXI scan as the Eddington limited flux. Equating the luminosity to the empirical Eddington luminosity of $L_{\mathrm{Edd}}=3.8 \times 10^{38} \mathrm{erg} \mathrm{s}^{-1}$ (Kuulkers et al. 2003), we find a distance of $d=7.3 \pm$ $0.5 \mathrm{kpc}$. Degenaar et al. (2013) derived a distance of $5 \mathrm{kpc}$, but this was done by extrapolating the peak flux of the 2012 burst without detecting PRE.

Using an energy release of burning He to $\mathrm{Ni}$ of $E_{\text {nuc }} \simeq$ $10^{18} \mathrm{erg} \mathrm{g}^{-1}$ and the above distance, we find the burst energy of $E_{\mathrm{b}}=\left(4.5_{-1.0}^{+1.6}\right) \times 10^{41} \mathrm{erg}$ in the observer frame. Part of the burst energy may escape the neutron star as a neutrino flux. We neglect it here, as it is expected to be minor for columns of $y \lesssim 10^{11} \mathrm{~g} \mathrm{~cm}^{-2}$ (Keek \& Heger 2011). During PRE, a substantial part of the burst energy may power a radiative wind (e.g., Weinberg et al. 2006). The fraction of the fluence during the PRE phase is $t_{\mathrm{PRE}} F_{\mathrm{Edd}} / \mathcal{F}_{\text {burst }}=0.13_{-0.10}^{+0.14}$. The total burst energy could, therefore, be larger by $\sim 10 \%$. Assuming a neutron star mass of $1.4 M_{\odot}$ and radius of $R=10 \mathrm{~km}$, which give a gravitational redshift of $1+z=1.31$, we find the ignition column:

$$
y_{\mathrm{ign}}=\frac{(1+z) E_{\mathrm{b}}}{4 \pi R^{2} E_{\mathrm{nuc}}}=\left(4.7_{-1.0}^{+1.6}\right) \times 10^{10} \mathrm{~g} \mathrm{~cm}^{-2} .
$$

5.2. Persistent Accretion

We have fit several models to the persistent flux (Section (3). When extrapolating the model to lower energies, a power law gives an unphysically large bolometric flux. The reflection model has a low-energy turn-over of the power law at $0.1 \mathrm{keV}$, but the turn-over is likely near the peak of the blackbody at an order of magnitude higher energy. Therefore, we prefer the bolometric flux obtained with the simpl model (Table 4). Multiplying the flux measured for Chandra spectrum 17543 by the MAXI scaling factor, we find a bolometric unabsorbed flux of $F_{\text {pers }}=(0.92 \pm 0.07) \times 10^{-10} \mathrm{erg} \mathrm{s}^{-1} \mathrm{~cm}^{-2}$. This is the time-averaged persistent flux between the bursts in 2012 and 2015. Using the flux of the first MAXI burst observation as a measure of the Eddington limit, we find $F_{\text {pers }} / F_{\text {Edd }}=(1.5 \pm 0.3) \times 10^{-3}$. 
Only a few LMXBs have shown bursts at such low persistent flux (e.g., Kaptein et al. 2000; Degenaar et al. 2010; Chenevez et al. 2012), and IGR J17062-6143 presents a rare instance of recurring bursts at low mass accretion rate (1RXS J171824.2-402934 is another example; in't Zand et al. 2009). The time between the triggers of the bursts in 2012 and 2015 is $3.3552 \mathrm{yr}$, and the ratio of the persistent and burst fluences is $\alpha=\mathcal{F}_{\text {pers }} / \mathcal{F}_{\text {burst }}=(1.4 \pm 0.4) \times 10^{2}$. Assuming $100 \%$ efficiency of converting gravitational energy to X-rays, a column of $y_{\text {acc }}=(2.7 \pm 0.4) \times 10^{10} \mathrm{~g} \mathrm{~cm}^{-2}\left(\frac{1.4 M_{\odot}}{M}\right)\left(\frac{10 \mathrm{~km}}{R}\right)$ was accreted. This includes the uncertainty in the distance. When comparing $y_{\text {acc }}$ to $y_{\text {ign }}$, we can avoid this uncertainty by simply taking

$\frac{y_{\text {acc }}}{y_{\text {ign }}}=\frac{\mathcal{F}_{\text {pers }}}{\mathcal{F}_{\text {burst }}} \frac{E_{\text {nuc }}}{G M / R}=0.73_{-0.14}^{+0.23}\left(\frac{1.4 M_{\odot}}{M}\right)\left(\frac{R}{10 \mathrm{~km}}\right)$,

which means that the two columns are consistent within $1.2 \sigma$. This confirms that the X-ray luminosity is indeed a good measure of the mass accretion rate in this system. Unfortunately, there were many instances when the source was not observed by any X-ray instrument for several hours, and we cannot verify that indeed no other burst occurred between 2012 and 2015. MAXI covered the source with at least one scan per 3 hours for $63 \%$ of the time, and similarly gaps are present in the coverage by Swift/BAT.

$y_{\text {ign }}$ is substantially smaller than typical for superbursts (Cumming et al. 2006), especially at low mass accretion rates (e.g., Kuulkers et al. 2010; Altamirano et al. 2012), where carbon ignition is expected to occur much deeper Cumming et al. 2006; Keek \& Heger 2011). Therefore, we classify the event as due to deep helium ignition. Models of pure helium accretors, however, predict $y_{\text {ign }}$ to be much larger at the observed mass accretion rate (Cumming et al. 2006). These models consider heating of the neutron star envelope by nuclear processes in the crust and neutrino cooling in the core. The maximum heating that this provides is insufficient to explain the observed $y_{\text {ign }}$, and additional "shallow" heating would be required to ignite helium at the observed depth (Brown \& Cumming 2009; Deibel et al. 2015, 2016). This burst from IGR J170626143 could indicate that shallow heating is active even at very low mass accretion rates. Alternatively, the accretion composition could include some hydrogen, the burning of which could heat the envelope and lower $y_{\text {ign }}$ to the observed value. Optical spectroscopic observations of the companion star could determine the presence of hydrogen.

\subsection{Reflection Signals}

The 2012 burst spectrum exhibited a strong emission line near $1 \mathrm{keV}$, which was suggested to be a fluorescent Fe $\mathrm{L}$ line originating at a distance of $\sim 10^{2} R_{\mathrm{g}}$ from the neutron star (Degenaar et al. 2013). We find an emission feature with similar energy and width in the XRT spectra of the 2015 burst. The normalization of the line decreases at the same rate as the blackbody flux, and the line during the 2012 burst roughly follows the same trend. This is a strong indication that the line is produced by reprocessed burst emission. High resolution
Chandra spectra of the persistent emission also exhibit emission features near $1 \mathrm{keV}$. Furthermore, the $N u S T A R$ persistent spectra clearly exhibit a broadened Fe $\mathrm{K} \alpha$ line and a Compton hump: the tell-tale signs of photoionized reflection (Degenaar et al. 2016). Therefore, we argue that the $1 \mathrm{keV}$ feature is similarly produced by reflection. During the bursts, the feature is accompanied by a soft excess, which may be the Bremsstrahlung continuum from reflection. Reflection models can describe the soft excess and part of the $1 \mathrm{keV}$ line, but residuals remain around the line both for the 2015 burst and the persistent spectra. The strength of the lines in the reflection spectrum is highly dependent on the composition (e.g., Madej et al. 2014) and the density of the reflecting material. Current reflection models are limited in this respect, which may be the cause of the imperfect description of the observed feature.

The reflection models suggest that the disk is highly ionized $(\log \xi \simeq 3)$ both during the burst and the persistent spectrum, even though the illuminating flux changes by orders of magnitude. During two superbursts, $\log \xi$ was observed to decrease over time (Ballantyne \& Strohmayer 2004; Keek et al. 2014b), but for several other sources the persistent $\log \xi$ is similarly large (e.g., Cackett et al. 2010; Degenaar et al. 2015; Iaria et al. 2016). As $\xi$ depends on both flux and density (Section 2.4), one explanation could be an increase in the density of the reflection site during the burst, for example if the burst strips the low-density upper layer from the disk. Low density material could also be present at the inner edge of a disk that is truncated by the neutron star's magnetic field (Ballantyne et al. 2012). The persistent flux could reflect off this material, whereas the burst flux may reflect off the disk. Another possibility is that the inner disk is overionized, such that it does not produce emission lines. Instead of the inner radius of the disk, $R_{\text {in }}$ would correspond to a location further out in the disk, where $\xi$ is sufficiently reduced to produce the reflection features. For stronger illumination, $R_{\text {in }}$ would then increase, whereas we see it decrease during the burst. Care must be taken, however, not to overinterpret the present results, because the uncertainties in $R_{\text {in }}$ are large, at least in part due to the limitations of the reflection models (Section 2.4).

\subsection{Burst Impact on Accretion Environment}

Both the persistent and burst spectra are welldescribed by a blackbody and a power law. The blackbody likely originates at the neutron star surface, which is heated by persistent accretion and by thermonuclear burning during the burst. The blackbody temperature demonstrates cooling in the burst tail, which is often cited as a defining characteristic of a Type I X-ray burst of thermonuclear origin. Over the course of a day, the blackbody flux decreases with time as $F_{\mathrm{bb}} \propto t^{-1.15 \pm 0.14}$. The power is just $1.1 \sigma$ smaller than the expected $\simeq 1.3$ for bursts that ignite at large column depths where cooling is dominated by ions (in't Zand et al. 2014a).

During the burst and the subsequent 11 days, the power law flux traces the changes in the blackbody flux. The power law is likely produced by reprocessing of the blackbody emission. We speculate that this is Comptonization in an optically thin plasma near the neutron star. We refer to it as a "corona", but it may be a bound- 
ary layer or accretion flow between a truncated disk and the neutron star. As no high-energy cut-off is detected in the $N u S T A R$ band, the electrons in the plasma must be hot: $k T_{\mathrm{e}} \gtrsim 10^{2} \mathrm{keV}$. During the PRE phase at the start of the burst, the $M A X I$ spectrum shows no sign of the power law, suggesting disruption of the corona by the burst. In the burst tail the corona may have reformed, since the power law returns with a similar $\Gamma$ and a slightly lower scattering fraction than in the persistent spectrum.

Fits with reflection models find an inner disk radius of $R_{\text {in }} \sim 10^{2} R_{\mathrm{g}}$ for the persistent emission, which suggests that the disk is truncated far from the neutron star (Degenaar et al. 2016), whereas in the burst tail the disk may have moved inwards to $R_{\text {in }} \sim 10^{1} R_{\mathrm{g}}$. Possibly, in the tail Poynting-Robertson drag brings the inner disk closer to the neutron star (Walker 1992; Ballantyne \& Everett 2005; Worpel et al. 2013, 2015). At the burst start a large $R_{\text {in }}$ is favored, and the effect of Poynting-Robertson drag may have been temporarily kept at bay by radiation pressure or an outflowing wind. However, the quality of the burst spectra is modest, and the uncertainty in $R_{\text {in }}$ is large.

Another quantity to consider is the reflection fraction, which at all times is substantial. For the persistent emission, a large $R_{\text {in }}$ and reflection fraction may be consistent if the disk is illuminated by a corona that extends at least to $R_{\text {in }}$, such that a substantial part of the power law flux is intercepted by the disk. In the burst the blackbody dominates the reflection signal instead of the power law. In the tail, the measured reflection fraction meets the expectations for a flat disk that extends to the neutron star (Lapidus \& Sunvaev 1985; Fujimoto 1988; He \& Keek 2016), and $R_{\text {in }}$ at this time is indeed consistent with no or a small gap between star and disk. At the burst start, however, the reflection fraction is substantially larger than unity (with a large error). This may require a steeply inclined reflection surface close to the star (He \& Keek 2016), but the measured $R_{\text {in }}$ is large. However, the large uncertainty of $R_{\text {in }}$ allows for a value of similar size as in the tail. Therefore, we favor the scenario where the disk is truncated at $R_{\text {in }} \sim 10^{2} R_{\mathrm{g}}$ during persistent accretion, whereas the impact of the burst brings the inner disk close to the neutron star, both at the burst start and in the tail.

During the burst, the behavior of the source is dominated by the cooling of the blackbody, until the total bolometric flux is reduced to the pre-burst persistent level. Over the course of a week, the spectrum returns to being dominated by the power law. At the end of the XRT observations, both $\Gamma$ and the scattering fraction are lower than the persistent values. Perhaps the corona has not fully recovered from the burst, which may take longer than for other bursting LMXBs, because of the low mass accretion rate. The influence of a burst on the hard tail has previously been inferred for a superburst (Keek et al. 2014a) and by stacking short bursts (Maccarone \& Coppi 2003; Chen et al. 2013; Ji et al. 2014). Interestingly, at $t \simeq 2 \times 10^{5} \mathrm{~s}$ the spectrum suddenly changes, and it takes several days to return to its previous behavior. The blackbody, which we attribute to the cooling neutron star, is strongly suppressed at this time, and the overall source flux is reduced. The flux reduction could be the result of an inner disk depleted by Poynting-Robertson drag. The restoration of the inner disk and the accretion flow might briefly block our view of the neutron star.

The limitations posed by the modest quality of the burst spectra and by the reflection models make it challenging to put firm constraints on the interaction of the burst and the accretion environment. It is however, clear that the burst has a strong influence on both the "corona" and the disk (for further discussion, see Ballantyne \& Everett 2005).

\subsection{Reprocessing and Anisotropic Emission}

We have derived the burst properties under the assumption of isotropic emission, which is not the case in LMXBs (Lapidus \& Sunvaev 1985; Fujimoto 1988; He \& Keek 2016). Now that we have a picture of the different emission and reprocessing sites in IGR J170626143 , we investigate how this affects the observed burst flux. Anisotropy factors for burst emission have only been calculated for disks that extend to the neutron star surface. The burst tail may be the closest to this situation. The measured reflection fraction of $0.39 \pm 0.05$ corresponds to a geometrically thin disk with an inclination angle of $39^{\circ} \pm 7^{\circ}$ (He \& Keek 2016), which is consistent with the absence of eclipses and dips, and is $1.3 \sigma$ larger than the $30^{\circ}$ that we assumed in Section 3.3. It implies an anisotropy factor of $\xi_{d}^{-1}=0.92 \pm 0.03$ for the direct blackbody emission (He \& Keek 2016), and the intrinsic direct neutron star flux is larger by $9 \%$ than observed. For truncated disks, blocking of the line of sight by the disk is smaller, and $\xi_{\mathrm{d}}^{-1}$ is even closer to unity.

We find that a scattering fraction $f_{\text {sc }}$ of the blackbody emission is reprocessed by a Comptonizing corona. If the corona has a spherical geometry that envelopes the neutron star, its anisotropy is similarly small as for the blackbody itself. Part of the blackbody is scattered from the line of sight, and the intrinsic neutron star emission is the sum of the observed blackbody and the Comptonized part. Indeed, the burst fit that includes the simpl model returns a blackbody flux that is lower by a factor $\left(1-f_{\mathrm{sc}}\right)$ than the blackbody flux from the phenomenological fit (Section 4.2) .7 The latter is, therefore, a good measure of the intrinsic isotropic burst emission. The anisotropy correction for the persistent flux may be similarly small.

At the start of the burst, we suspect that the disk may not be flat. The anisotropy is strongly dependent on the disk geometry (He \& Keek 2016), and in principle a large correction could be needed: $\xi_{\mathrm{d}}^{-1} \sim 0.1$. This would have important consequences for the derived quantities such as the distance and $y_{\text {ign }}$. Fortunately, the Comptonizing corona is absent at the burst start, and we can regard all flux to be from the blackbody and its reflection, the sum of which may underestimate the intrinsic burst emission by at most tens of percents (He \& Keek 2016).

\section{CONCLUSIONS AND OUTLOOK}

We performed a time-resolved spectral analysis of the 2015 Type I X-ray burst from IGR J17062-6143 observed with MAXI and Swift (Negoro et al. 2015; Iwakiri et al. 2015). The burst is of exceptional duration, both because its deep ignition providing a long decay timescale

\footnotetext{
7 The power law in Section 4.1 fits mostly to the soft excess, which is described by reflection in Section 4.2 whereas the simpl component fits to the hard tail.
} 
of $\tau \simeq 21$ minutes, and because the low persistent flux of $(1.5 \pm 0.3) \times 10^{-3} F_{\text {Edd }}$ allows the burst cooling to be detectable for close to a day. Analysis of the persistent flux observations with $M A X I$, Chandra, and $N u S$ $T A R$ shows that the column accreted since the previous burst in 2012 is consistent with the ignition column of $y_{\text {ign }}=\left(4.7_{-1.0}^{+1.6}\right) \times 10^{10} \mathrm{~g} \mathrm{~cm}^{-2}$ derived for the 2015 burst. The burst onset exhibits radius expansion, and the tail describes a straight power law: $F \propto t^{-1.15 \pm 0.14}$. We categorize this event as an intermediate duration burst from deep helium burning, and it may be the longest helium burst observed to date.

Both the burst and persistent spectra are well described by a blackbody and a power law (see also Degenaar et al. 2016). The persistent NuSTAR spectra exhibit clear evidence of photoionized reflection of the power law (Degenaar et al. 2016). The Swift/XRT burst spectra exhibit a soft excess and an emission line at $1 \mathrm{keV}$ that also suggest reflection of the burst off the disk. We investigate a description of the spectra that includes burst reflection and a Comptonized component (instead of the power law). The Comptonized part is missing at the peak of the burst, and has returned in the tail: possibly a Comptonizing corona is temporarily disrupted in the brightest phase of the burst. The reflection models find the disk to be highly ionized at all times, but the reflection location moves substantially closer to the neutron star during the burst from $R_{\text {in }} \simeq 2 \times 10^{2} R_{\mathrm{g}}$ to $\simeq 14 R_{\mathrm{g}}$. Poynting-Robertson drag exerted by the burst could increase the inflow of matter (Walker 1992; Worpel et al. 2013, 2015).
The Neutron Star Interior Composition Explorer (NICER; Gendreau et al. 2012) to be launched in 2017 will host a 17 times larger effective area at $1 \mathrm{keV}$ than Swift/XRT, whereas ATHENA's (Barcons et al. 2015) Wide Field Imager (WFI; Meidinger et al. 2014) promises a 131 times larger effective area (launch in 2028). Future observations of intermediate duration bursts with these instruments will, therefore, provide a detailed view of the interesting processes that we glimpsed with $M A X I$ and Swift. Moreover, NICER, ATHENA, and a mission like the Large Observatory for X-Ray Timing (LOFT; Feroci et al. 2014; in 't Zand et al. 2015) with a collecting area of $\sim 8 \mathrm{~m}^{2}$ will also be able to detect interaction with the accretion environment during the frequent short bursts (Keek et al. 2016). This opens up a new avenue to study accretion processes. We saw that the already complex reflection models will need to further take into account a wider range of compositions and densities to take full advantage of such new X-ray burst observations (Ballantyne 2004; García et al. 2016).

LK is supported by NASA under award number NNG06EO90A. LK thanks the International Space Science Institute in Bern, Switzerland for hosting an International Team on X-ray bursts. This work benefited from events supported by the National Science Foundation under Grant No. PHY-1430152 (JINA Center for the Evolution of the Elements). This research has made use of MAXI data provided by RIKEN, JAXA and the $M A X I$ team. We thank the Swift observatory for performing the observations described in this paper.

\section{REFERENCES}

Altamirano, D., et al. 2012, MNRAS, 426, 927

Arnaud, K. A. 1996, in Astronomical Society of the Pacific Conference Series, Vol. 101, Astronomical Data Analysis Software and Systems V, ed. G. H. Jacoby \& J. Barnes, 17 Ballantyne, D. R. 2004, MNRAS, 351, 57

Ballantyne, D. R., \& Everett, J. E. 2005, ApJ, 626, 364 Ballantyne, D. R., Purvis, J. D., Strausbaugh, R. G., \& Hickox, R. C. 2012, ApJ, 747, L35

Ballantyne, D. R., \& Strohmayer, T. E. 2004, ApJ, 602, L105

Barcons, X., Nandra, K., Barret, D., den Herder, J.-W., Fabian, A. C., Piro, L., Watson, M. G., \& the Athena Team. 2015, Journal of Physics Conference Series, 610, 012008

Barthelmy, S. D., et al. 2005, Space Sci. Rev., 120, 143

Belian, R. D., Conner, J. P., \& Evans, W. D. 1976, ApJ, 206, L135

Brown, E. F., \& Cumming, A. 2009, ApJ, 698, 1020

Burrows, D. N., et al. 2005, Space Sci. Rev., 120, 165

Cackett, E. M., et al. 2010, ApJ, 720, 205

Canizares, C. R., et al. 2005, PASP, 117, 1144

Chen, Y.-P., Zhang, S., Zhang, S.-N., Ji, L., Torres, D. F., Kretschmar, P., Li, J., \& Wang, J.-M. 2013, ApJ, 777, L9

Chenevez, J., et al. 2012, The Astronomer's Telegram, 4050

Churazov, E., et al. 2007, A\&A, 467, 529

Cornelisse, R., et al. 2003, A\&A, 405, 1033

Cumming, A., Macbeth, J., in 't Zand, J. J. M., \& Page, D. 2006, ApJ, 646, 429

D'Angelo, C. R., Fridriksson, J. K., Messenger, C., \& Patruno, A. 2015, MNRAS, 449, 2803

Dauser, T., García, J., Parker, M. L., Fabian, A. C., \& Wilms, J. 2014, MNRAS, 444, L100

Dauser, T., Wilms, J., Reynolds, C. S., \& Brenneman, L. W. 2010, MNRAS, 409, 1534

Degenaar, N., Miller, J. M., Chakrabarty, D., Harrison, F. A., Kara, E., \& Fabian, A. C. 2015, MNRAS, 451, L85

Degenaar, N., Miller, J. M., Wijnands, R., Altamirano, D., \& Fabian, A. C. 2013, ApJ, 767, L37
Degenaar, N., Pinto, C., Miller, J. M., Wijnands, R., Altamirano, D., Paerels, F., Fabian, A. C., \& Chakrabarty, D. 2016, MNRAS

Degenaar, N., et al. 2010, MNRAS, 404, 1591

Deibel, A., Cumming, A., Brown, E. F., \& Page, D. 2015, ApJ, 809, L31

Deibel, A., Meisel, Z., Schatz, H., Brown, E. F., \& Cumming, A. 2016, ArXiv e-prints

Feroci, M., et al. 2014, in Society of Photo-Optical

Instrumentation Engineers (SPIE) Conference Series, Vol. 9144, Society of Photo-Optical Instrumentation Engineers (SPIE) Conference Series, 2

Fujimoto, M. Y. 1988, ApJ, 324, 995

Galloway, D. K., Muno, M. P., Hartman, J. M., Psaltis, D., \& Chakrabarty, D. 2008, ApJS, 179, 360

García, J., Dauser, T., Reynolds, C. S., Kallman, T. R., McClintock, J. E., Wilms, J., \& Eikmann, W. 2013, ApJ, 768, 146

García, J., et al. 2014, ApJ, 782, 76

García, J. A., Fabian, A. C., Kallman, T. R., Dauser, T., Parker, M. L., McClintock, J. E., Steiner, J. F., \& Wilms, J. 2016, MNRAS, 462, 751

Gehrels, N., et al. 2004, ApJ, 611, 1005

Gendreau, K. C., Arzoumanian, Z., \& Okajima, T. 2012, in Society of Photo-Optical Instrumentation Engineers (SPIE) Conference Series, Vol. 8443

Grindlay, J., Gursky, H., Schnopper, H., Parsignault, D. R.,

Heise, J., Brinkman, A. C., \& Schrijver, J. 1976, ApJ, 205, L127

Harrison, F. A., et al. 2013, ApJ, 770, 103

He, C.-C., \& Keek, L. 2016, ApJ, 819, 47

Huenemoerder, D. P., et al. 2011, AJ, 141, 129

Iaria, R., et al. 2016, ArXiv e-prints

in 't Zand, J. J. M., Galloway, D. K., \& Ballantyne, D. R. 2011, A\&A, 525, A111 
in 't Zand, J. J. M., Jonker, P. G., \& Markwardt, C. B. 2007, A\&A, 465, 953

in 't Zand, J. J. M., et al. 2015, ArXiv e-prints

in't Zand, J. J. M., Cumming, A., Triemstra, T. L., Mateijsen, R. A. D. A., \& Bagnoli, T. 2014a, A\&A, 562, A16

in't Zand, J. J. M., Jonker, P. G., Bassa, C. G., Markwardt, C. B., \& Levine, A. M. 2009, A\&A, 506, 857

in't Zand, J. J. M., Keek, L., \& Cavecchi, Y. 2014b, A\&A, 568, A69

Ivarsen, K., et al. 2012, GRB Coordinates Network, 13390

Iwakiri, W., Keek, L., Serino, M., Strohmayer, T., in't Zand, J., Kuulkers, E., \& Sakamoto, T. 2015, The Astronomer's Telegram, 8253

Ji, L., Zhang, S., Chen, Y., Zhang, S.-N., Torres, D. F., Kretschmar, P., \& Li, J. 2014, ApJ, 782, 40

Kalberla, P. M. W., Burton, W. B., Hartmann, D., Arnal, E. M., Bajaja, E., Morras, R., \& Pöppel, W. G. L. 2005, A\&A, 440, 775

Kaptein, R. G., in't Zand, J. J. M., Kuulkers, E., Verbunt, F., Heise, J., \& Cornelisse, R. 2000, A\&A, 358, L71

Keek, L., \& Ballantyne, D. R. 2016, MNRAS, 456, 2722

Keek, L., Ballantyne, D. R., Kuulkers, E., \& Strohmayer, T. E. 2014a, ApJ, 789, 121

—. 2014b, ApJ, 797, L23

Keek, L., \& Heger, A. 2011, ApJ, 743, 189

Keek, L., \& in 't Zand, J. J. M. 2008, in Proceedings of the 7th INTEGRAL Workshop. 8 - 11 September 2008 Copenhagen, Denmark. Online at http://pos.sissa.it/cgi-bin/reader/conf.cgi?confid $=67$, p. 32

Keek, L., Wolf, Z., \& Ballantyne, D. R. 2016, ApJ, 826, 79

Kuulkers, E., den Hartog, P. R., in 't Zand, J. J. M., Verbunt, F. W. M., Harris, W. E., \& Cocchi, M. 2003, A\&A, 399, 663

Kuulkers, E., et al. 2010, A\&A, 514, A65+

Lapidus, I. I., \& Sunyaev, R. A. 1985, MNRAS, 217, 291

Linares, M., et al. 2012, ApJ, 760, 133

Maccarone, T. J., \& Coppi, P. S. 2003, A\&A, 399, 1151

Madej, O. K., García, J., Jonker, P. G., Parker, M. L., Ross, R., Fabian, A. C., \& Chenevez, J. 2014, MNRAS, 442, 1157

Maraschi, L., \& Cavaliere, A. 1977, in Highlights in Astronomy, ed. E. A. Müller, Vol. 4 (Reidel, Dordrecht), 127

Matsuoka, M., et al. 2009, PASJ, 61, 999
Meehan, S., Hanlon, L., Topinka, M., \& Kubanek, P. 2012, GRB Coordinates Network, 13396

Meidinger, N., Nandra, K., Plattner, M., Porro, M., Rau, A., Santangelo, A. E., Tenzer, C., \& Wilms, J. 2014, in Proc. SPIE, Vol. 9144, Space Telescopes and Instrumentation 2014: Ultraviolet to Gamma Ray, 91442J

Mihara, T., et al. 2011, PASJ, 63, S623

Moretti, A., et al. 2005, in Society of Photo-Optical Instrumentation Engineers (SPIE) Conference Series, Vol. 5898, UV, X-Ray, and Gamma-Ray Space Instrumentation for Astronomy XIV, ed. O. H. W. Siegmund, 360-368

Negoro, H., et al. 2015, The Astronomer's Telegram, 8241

-. 2016, PASJ, 68, S1

Pinto, C., Kaastra, J. S., Costantini, E., \& Verbunt, F. 2010, A\&A, 521, A79

Remillard, R. A., \& Levine, A. M. 2008, The Astronomer's Telegram, 1853

Ricci, C., Beckmann, V., Carmona, A., \& Weidenspointner, G. 2008, The Astronomer's Telegram, 1840

Roming, P. W. A., et al. 2005, Space Sci. Rev., 120, 95

Schlegel, D. J., Finkbeiner, D. P., \& Davis, M. 1998, ApJ, 500, 525

Steiner, J. F., Narayan, R., McClintock, J. E., \& Ebisawa, K. 2009, PASP, 121, 1279

Strohmayer, T. E., \& Brown, E. F. 2002, ApJ, 566, 1045

Sugizaki, M., et al. 2011, PASJ, 63, S635

Walker, M. A. 1992, ApJ, 385, 642

Weinberg, N. N., Bildsten, L., \& Schatz, H. 2006, ApJ, 639, 1018

Weisskopf, M. C., Tananbaum, H. D., Van Speybroeck, L. P., \&

O'Dell, S. L. 2000, in Proc. SPIE, Vol. 4012, X-Ray Optics, Instruments, and Missions III, ed. J. E. Truemper \& B. Aschenbach, 2-16

Willingale, R., Starling, R. L. C., Beardmore, A. P., Tanvir, N. R., \& O'Brien, P. T. 2013, MNRAS, 431, 394

Wilms, J., Allen, A., \& McCray, R. 2000, ApJ, 542, 914

Woosley, S. E., \& Taam, R. E. 1976, Nature, 263, 101

Worpel, H., Galloway, D. K., \& Price, D. J. 2013, ArXiv e-prints -. 2015, ApJ, 801, 60 